\section{Check for updates}

Cite this: Mater. Adv., 2021, 2, 7799

Received 1st September 2021 Accepted 26th October 2021

DOI: $10.1039 / \mathrm{d} 1 \mathrm{ma} 00789 \mathrm{k}$

rsc.li/materials-advances

\title{
Functional liquid metal nanoparticles: synthesis and applications
}

\author{
Cerwyn Chiew, ${ }^{a}$ Maria J. Morris ${ }^{a}$ and Mohammad H. Malakooti (D) *ab
}

\begin{abstract}
Room temperature liquid metals are an emerging class of functional materials with applications in a variety of soft intelligent systems. In recent years, efforts have been made to integrate liquid metal alloys such as eutectic gallium-indium (EGaln) and eutectic gallium-indium-tin (Galinstan) into wearable electronics, soft robotics, and biomedical devices. One main methodology is reducing the size of the liquid metal (LM) droplets to the micro and nanoscale level. In this review, we present an overview of the recent progress in synthesizing liquid nanoparticles with a focus on the role of processing parameters on their structure and surface properties. Next, the liquid metal nanoparticles are discussed as the functional units in polymer nanocomposites with enhanced electrical and thermal properties. Ultimately, the applications of LM nanoparticles and nanocomposites are presented, followed by a brief outlook on the challenges and future developments of advanced liquid metal material systems.
\end{abstract}

\section{Introduction}

The fluidic and metallic properties of room temperature liquid metal alloys have attracted numerous studies, in which they are incorporated as microfluidic-based stretchable conductors for soft and reconfigurable electronics, ${ }^{1,2}$ and cooling fluids for miniature electronics. ${ }^{3,4}$ To serve these purposes, EGaIn $(75 \%$ gallium and $25 \%$ indium by weight) and Galinstan $(68.5 \%$ gallium, $21.5 \%$ indium and $10 \%$ tin by weight) are the two widely utilized gallium $(\mathrm{Ga})$ based room temperature LMs to date because of their low toxicity, ${ }^{1,5}$ low viscosity $(2 \times$ that of water) ${ }^{6}$ high electrical conductivity, ${ }^{7}$ and high thermal conductivity. ${ }^{3,6}$ With the recent adaptations of novel processing techniques, bulk LM alloys have also been facilely engineered as multifunctional nanocomposites ${ }^{8,9}$ or functional nanoparticles ${ }^{10}$ for diverse applications.

A unique feature of the Ga-based LM alloys is their thin (1-3 nm) passivating Ga oxide interface which encapsulates the LM alloy droplets as a core-shell structure, even in trace amounts of oxygen ${ }^{11,12}$ (Fig. 1). This oxide interface exhibits viscoelastic behaviors, ${ }^{13}$ regulates the surface tension of the $\mathrm{Ga}$ LM alloys, and enables wettability with various surfaces. ${ }^{14,15}$ These features have enabled bulk EGaIn to be used as a soft cone-shaped microelectrode to accurately measure the electrical $^{16,17}$ and thermoelectric ${ }^{18-20}$ properties of thin organic nanomaterials. Moreover, the reactive oxide skin on the bulk

\footnotetext{
${ }^{a}$ Department of Mechanical Engineering, University of Washington, Seattle, WA 98195, USA.E-mail: malakoot@uw.edu

${ }^{b}$ Institute for Nano-Engineered Systems, University of Washington, Seattle, WA 98195, USA
}

Ga-based liquid metal alloys has been taken advantage of as a catalyst for growing carbon nanotubes, ${ }^{21}$ thin films of inorganic thermoelectric materials, ${ }^{22}$ and novel $2 \mathrm{D}$ nanomaterials with high piezoelectric conversion. ${ }^{23,24}$ When this bulk LM is downsized to nanodroplets, a large surface area of the oxide layer is created, forming the primary stabilizing interface for the synthesized LM micro or nanoparticles (NPs) in various media. ${ }^{25,26}$ Furthermore, the chemical reactivity of the Ga oxide layer provides a customizable platform for targeted surface

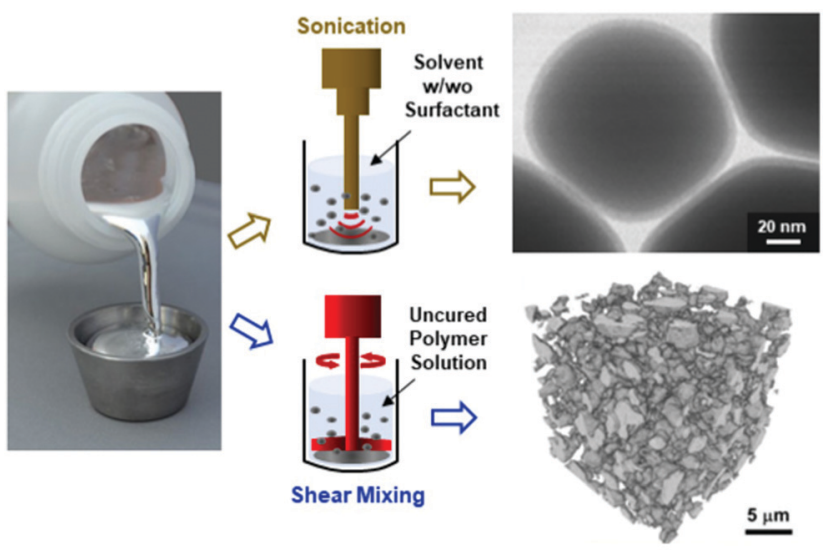

Fig. 1 Processing liquid metal nanoparticles (LMNPs) via high intensity sonication and shear mixing. Left: Image of bulk liquid metal being poured into a cup (Credit: Indium Corporation). Top right: Transmission electron microscopy (TEM) image of LM nanoparticles synthesized via sonication with thiol ligands. Reproduced with permission, ${ }^{29}$ copyright 2016 , Wiley-VCH. Bottom right: 3D nano-CT image of LM particles produced by emulsion shearing in uncured polymer. Reproduced with permission, ${ }^{30}$ copyright 2019 , Wiley-VCH. 
chemical functionalization or modification in order to tailor the nanoparticles' morphology and functional properties such as $\mathrm{pH}$ resistance, temperature changes, photothermal conversion, and electrical properties. ${ }^{10,27,28}$

The Ga oxide layer on the bulk liquid metal can be easily ruptured by applying surface stress. Once ruptured, it instantaneously reforms to stabilize the high surface tension $\left(\sim 0.5 \mathrm{~N} \mathrm{~m}^{-1}\right)$ of the LM. Thus, when a continuous mechanical stress is applied, the oxide interface of the newly formed LM droplets will be repeatedly ruptured and reformed, allowing the bulk LM to be rapidly downsized from the macro to micro and nanoscale. Some of the most common techniques to create micro/ nanoscale LM particles include the sonication of bulk LM in a solvent using an ultrasonic probe ${ }^{31,32}$ (Fig. 1) or an ultrasonic bath. $^{25,33}$ The in situ formation of LM microparticles in polymers or solvents has been done through the mechanical shear forces between these two liquid phases. For this purpose, bladeless planetary shear mixers ${ }^{33-35}$ and overhead stirrers with impellers are chosen to provide more intense mixing. ${ }^{30,36,37}$ To create a homogeneous LM polymer nanocomposite, one or more combinations of these methods are preferred. ${ }^{38,39}$ The shear-based synthesis methods are attractive because of their much facile, scalable, and rapid processes to create LM particles with sizes ranging from hundreds of microns to hundreds of nanometers. Ultrasonication methods, on the other hand, provide high mechanical energy via acoustic waves to rapidly downsize bulk LM to a nanoscale level and at the same time enable surface functionalization (i.e., surface in situ polymerization, organic or inorganic material coatings) or chemical modifications on the generated LM nanoparticles.

Reducing the size of LM droplets is desirable because it can decrease the freezing and melting points of its liquid core. For instance, the freezing point of EGaIn decreases from -5.9 to $-84.1{ }^{\circ} \mathrm{C}$ while the melting point decreases from 17.8 to $-25.6{ }^{\circ} \mathrm{C} .{ }^{30}$ Similarly, the following supercooling property is also found in the Field's metal (i.e., eutectic bismuth-indiumtin alloy) based LM nanoparticles where their suppressed freezing temperature is closer to room temperature. ${ }^{37,40,41}$ As a result, these supercooled LM nanoparticles can be used to create low temperature compliant thermal interfaces ${ }^{30}$ and heat-free electronic soldering materials. ${ }^{37,41}$ Also, in comparison to macroscale LM, nanoscale LM droplets exhibit higher photothermal conversion efficiency for aiding intracellular drug delivery $^{28,42-44}$ and lower chemical reactivity with metals ${ }^{45,46}$ while capable of increasing the thermal conductivity of traditional cooling fluids. ${ }^{47}$ When LM nanoparticles are embedded in nanocomposites, their size and volume fractions are tailorable parameters that determine the final elastic and functional (i.e., dielectric and thermal) properties of the composite. ${ }^{39,48,49}$ For instance, liquid metal composites with embedded nanoscale LM particles have larger dielectric constants and stiffness, enabling soft dielectric and thermal actuators to generate higher actuation forces. $^{38,39,50}$

The dominant surface area of the oxide interface found among nanoscale LM particles also reduces the interfacial surface tension for improved surface adhesion or wetting. This feature enables LM nanoparticle inks to form high resolution conductive traces printed using different additive manufacturing techniques (i.e., inkjet printing, nozzle dispensing, and ballpoint writing) for stretchable and reconfigurable interconnections. ${ }^{29,51-54}$ Despite this, the rigid and insulative oxide interface of the LM nanoparticles pose a challenge to create conductive traces. ${ }^{55-57}$ Several techniques ranging from mechanical, ${ }^{51,52,58}$ thermal, ${ }^{59}$ and laserbased $^{60,61}$ activations exist for coalescing LM nanoparticles. However, the ideal sintering strategy for LM nanoparticles will depend on the encapsulating material, interfacial surface modifications or functionalization, size of the dispersed LM nanoparticles, and desired resolution of the conductive traces.

Here, we will provide a comprehensive overview of recent advances in the synthesis and applications of liquid metal nanoparticles (LMNPs) with an emphasize on the relationship between the processing parameters and properties and their applications. With the emergence of liquid metal alloys in nanotechnology throughout the past few years, the number of research groups studying this topic has been steadily increasing. ${ }^{8,10}$ This has led to many research articles, perspectives, and review papers. Previous reviews on liquid metal nanomaterials have summarized the fabrication methods and applications of this class of nanoscale functional materials with distinct perspectives. For example, Song et $a .^{62}$ holistically covers the top-down and bottom-up fabrication methods of LM nanoparticles. A review paper by Lin et al. ${ }^{63}$ provides a broad overview of producing Ga-based LM particles with size ranging from millimeters to nanometers. Li et al. ${ }^{64}$ provided a focused progress report on the applications of LMNPs in biomedicine, while Liu et al. ${ }^{27}$ discussed the various surface engineering strategies conducted on LMNPs with a core-shell structure. Our timely review article is centered on the synthesis of free standing and embedded liquid metal nanoparticles. In particular, ultrasonication and mechanical shear mixing are discussed in detail as the two common scalable synthesis techniques for the fabrication of LMNPs. We provide a comprehensive analysis of processing parameters (time, power, solvent, surfactant concentrations and temperature) to create multifunctional liquid metal nanodroplets and nanocomposites with engineered surface/interface properties. In addition to the room temperature liquid metal droplets, the synthesis and applications of supercooled LMNPs such as eutectic bismuthindium-tin alloy are also discussed to highlight similarities in the processing methods and applications. After providing insights on the applications of nanoscale LM particles in printed conductors, wearable energy harvesting, and nanomedicine, we discussed the current challenges and future directions of this field.

\section{Synthesis methods of LM nanoparticles}

\subsection{Mechanical shearing in solvents}

Emulsion-based processing methods using an overhead stirrer and immersion mixers are rapid, scalable, temporally controllable, and facile approaches for synthesizing LM micro/nanodroplets. 
To generate nanoscale LM particles by immersion mixing, a large rotational velocity in a low viscosity mixing medium (bulk LM in ethanol) is needed. The immersed rotating blade facilitates the mixing process by applying the shear $(\gamma)$, centrifugal $\left(F_{\mathrm{c}}\right)$, and drag $\left(F_{\mathrm{d}}\right)$ forces along with the gravitational force $\left(F_{\mathrm{g}}\right)$ to continuously rupture the oxide shell of the large droplets (Fig. 2a). ${ }^{36}$ These combined forces cause bulk liquid metal to be sheared into micro or nano droplets.

During immersion mixing, the generated nanoscale coreshell LM particles can also have surface functionalization. An early study demonstrated this by shearing liquids into complex particles, a process termed SLICE, where large shearing forces are applied on bulk LM in an organic solvent with added surfactant (acetic acid in diethylene glycol). ${ }^{36,37}$ During this process, a thin, secondary complex acetate shell forms over the oxide shell of the LM particles. This additional outer layer prevents the newly formed LM particles from recoalescing, enabling a large quantity of nanoscale droplets to be produced with controllable polydispersity and suppressing the freezing point of the nanodroplets by providing a thermodynamic barrier against the solidification of their liquid core. ${ }^{41,65}$ The Field's metal nanodroplets, formed by immersion mixing, are also referred to as undercooled liquid metal core-shell particles, where the suppressed freezing point near room temperature is attractive for heat-free soldering in printed electronics. ${ }^{37,41}$ To demonstrate this property, when the Field's metal LM coreshell nanoparticles are sandwiched and ruptured by compression between gold laminates, the exuded undercooled LM cores recoalesce before immediately solidifying to form a solid mechanical bond between the gold sheets (Fig. 2b).

Immersion mixing of bulk LM also allows for repeatable control of the polydispersity and sizes of the formed LM droplets. For example, at a low shearing speed, the immersion mixing of bulk EGaIn in ethanol can synthesize highly polydisperse LM droplets. When the size distribution of the synthesized LM droplets has a ratio of largest to smallest particle diameter ranging from $1 / 7$ to $1 / 3$, these LM particles can be conformally jammed onto a highly textured soft biological surface (rose petals) and sintered via phase change (liquid to solid) to form replicas of the traced bio features as shown in Fig. 2c. ${ }^{66}$ As a result, this high polydispersity ensures that the average feature size of the replica $\left(\mathrm{a}_{2}{ }^{\prime}\right)$ formed by phase changed undercooled LM nanoparticles resembles the feature size of the original petal $\left(a_{2}\right)$ indicated in Fig. $2 d$.

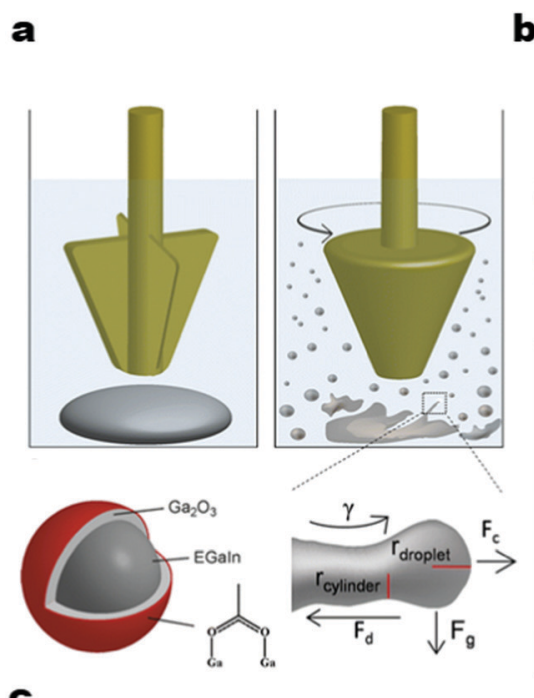

C

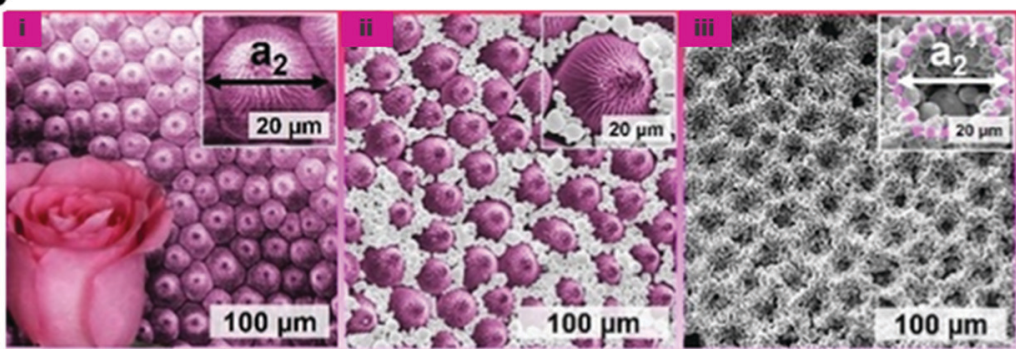

b
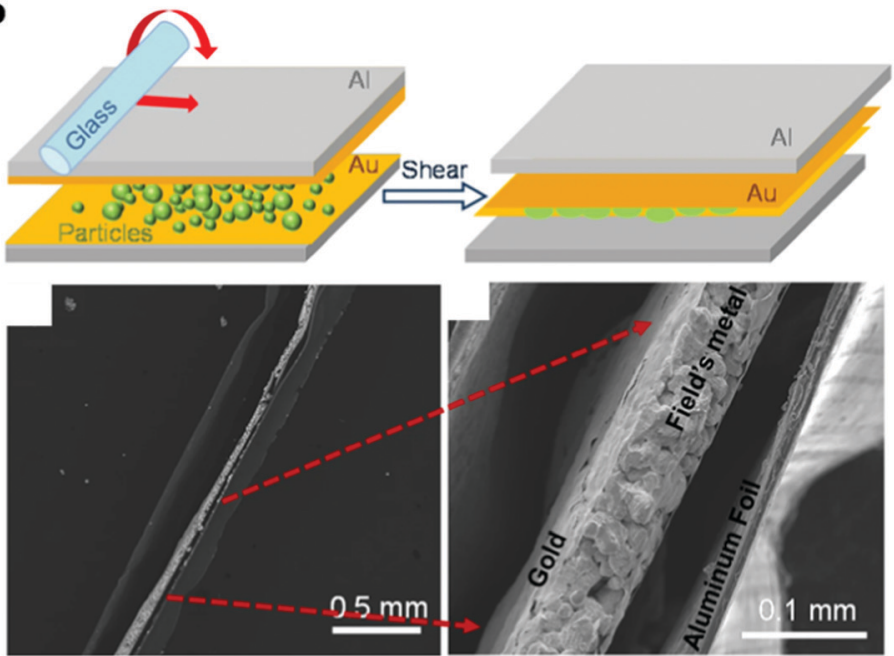

d

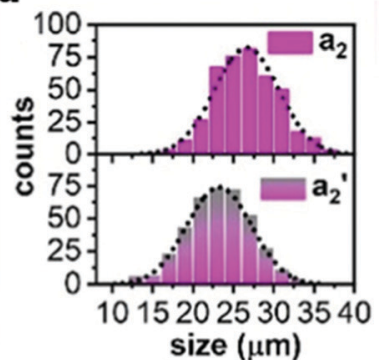

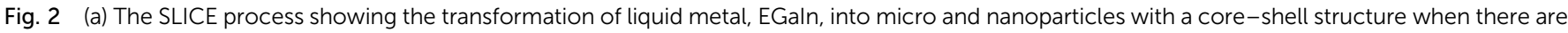

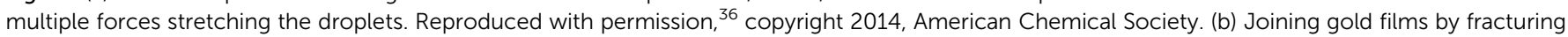

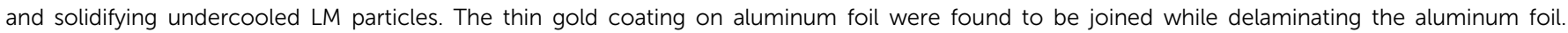

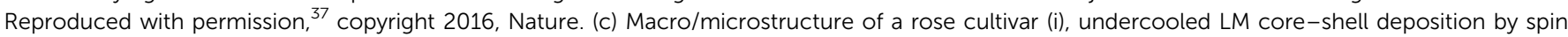

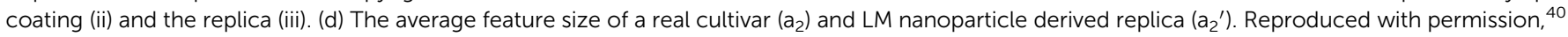
copyright 2020, Wiley-VCH. 


\subsection{Sonication-based synthesis}

To form a high concentration of nanoscale (i.e., $<1 \mu \mathrm{m}) \mathrm{LM}$ droplets, the passivating oxide shell of the LM droplets must be ruptured at a faster rate than it is reformed. Mechanical shearing sometimes cannot apply a sufficient rate of applied stress to produce LM nanoparticles in high yields. This leads to the use of probe-based ultrasonication methods which can create continuous or pulsed acoustic cavitation to rapidly break down large liquid metal droplets into micro-/nanoscale particles. Intense shear mixing of bulk LM with an adsorbate (polyvinylpyrrolidone or thiols) is only capable of slicing the LM alloys down to mostly the micron scale, whereas a sonication-assisted process can produce LM nanodroplets with narrower size distribution and as small as several tenths of orders of nanometers. ${ }^{32,51,56}$ Also, the use of ultrasonication to produce LM nanodroplets is beneficial because it reduces the amount of surfactants needed and consumes less energy than the shear-based synthesis process. ${ }^{67}$

Increasing the power and duration of the sonication will reduce the size of the LM nanoparticles until the solution reaches an equilibrium. Yamaguchi et al. ${ }^{31}$ illustrated this behavior for $\mathrm{Ga}$ particles and the relationship between the delivered power and the size of the particles (Fig. 3a). However, sonication without a surfactant in ethanol often results in LM nanodroplets with a highly polydisperse size, undefined spherical shape (textured, oblong and deformed spheres), and short-term dispersion stability as shown in Fig. $3 b^{25,68}$ The thin passivating oxide shell of the LM nanodroplets can be weakened by various chemical stimuli (acidic, basic, and highly oxygenated surroundings) and often require additional interfacial reinforcement to prevent the LM nanodroplets from recoalescing. During sonication, high energy acoustic cavitation exposes the reactive LM alloy core, causing it to actively bind with the self-assembling ligands (surfactants) while suppressing the formation of Ga oxide. As a result, the synthesized LM nanodroplets have a more rigid and chemically robust protective interface granted by the thiol layers. For example, well-formed and predominantly spherical LM particles are generated when bulk LM is sonicated in both 3-mercapto- $N$ nonylpropionamide (1ACT9) and 1-dodecanethiol (C12) ethanolic solutions (Fig. 3c). Comparing these two thiol ligands, the LM nanoparticles generated with 1ACT9 as the surfactant result in a better yield of spherical nanoparticles.

When the oxide shell is replaced by more resilient ligands, the formed nanoparticles will not recoalesce as easily and are more resistant to surrounding changes in $\mathrm{pH}$ or temperature. Nevertheless, regulating the acidity, temperature, and processing
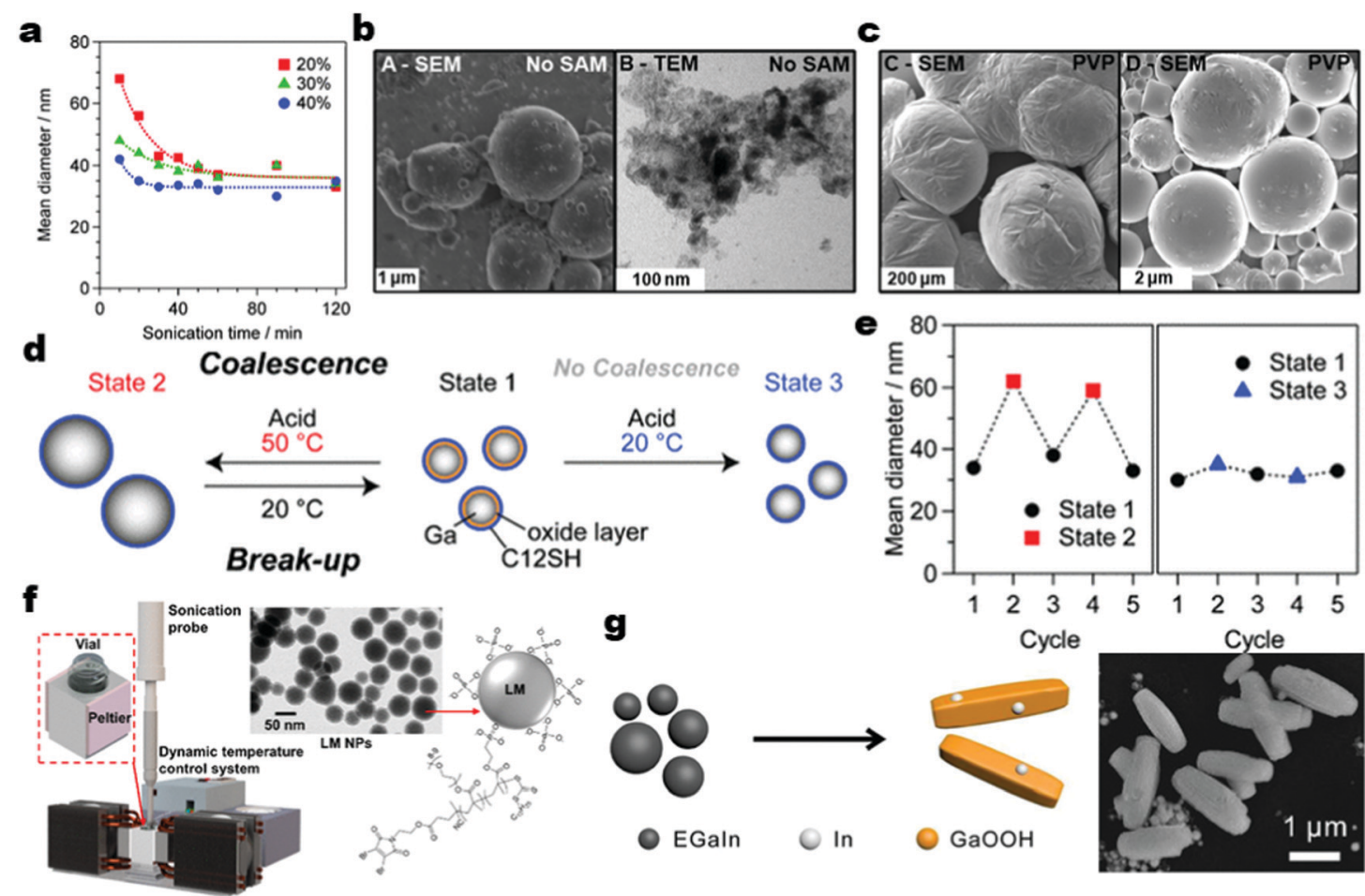

g
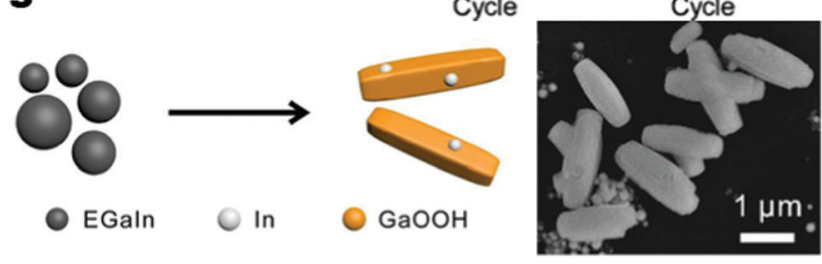

Fig. 3 (a) Mean diameters of the Ga nanoparticles as a function of ultrasonication time at different ultrasonication powers at $20{ }^{\circ} \mathrm{C}$. Reproduced with permission, ${ }^{31}$ copyright 2015, Wiley-VCH. (b) The EGaln droplets produced without thiol self-assembly and (c) the EGaln droplets produced with 1 ATC9 and $\mathrm{C} 12$ thiol ligands. Reproduced with permission, ${ }^{25}$ copyright 2011, American Chemical Society. (d) Reversibly controlling the size of Ga NPs. The precursor Ga NPs were ultrasonicated in the presence of $\mathrm{HCl}$ and $\mathrm{C} 12 \mathrm{SH}$ in 2-propanol at $50{ }^{\circ} \mathrm{C}$ (state $1 \rightarrow$ state 2 ) or $20{ }^{\circ} \mathrm{C}$ (state $1 \rightarrow$ state 3 ), and then again at $20^{\circ} \mathrm{C}$ for $20 \mathrm{~min}$ (state 2 or $3 \rightarrow$ state 1 ) and (e) the mean diameters of the Ga NPs in states $1-3$. Reproduced with permission, ${ }^{31}$ Copyright 2015 , Wiley-VCH. (f) The setup of dynamic temperature control for maintaining low sonication temperature when synthesizing LM nanodroplets with bPEG attachment. Reproduced with permission, ${ }^{69}$ copyright 2020 , American Chemical Society. (g) EGaln NP shape transformation and dealloying process with the SEM image of the shape transformed EGaln NP. Reproduced with permission, ${ }^{70}$ copyright 2021 , Wiley-VCH. 
time can provide reversible size control of the synthesized liquid metal nanoparticles. ${ }^{31}$ For example, $35 \mathrm{~nm}$ liquid Ga particles can be synthesized by sonication at low temperatures $\left(20^{\circ} \mathrm{C}\right)$ and at high temperatures $\left(50{ }^{\circ} \mathrm{C}\right.$ ) (state 1 to 2$)$ in the presence of dodecanethiol (C12SH) as the surfactant (Fig. 3d and e). Also, the size of these liquid $\mathrm{Ga}$ nanoparticles with $\mathrm{C12SH}$ as the surfactant is reversible. To demonstrate this, the Ga nanoparticles with a mean size of $35 \mathrm{~nm}$ are unchanged in the presence of acid when sonicated at a low temperature $\left(20{ }^{\circ} \mathrm{C}\right)$ because of the surface stabilization by $\mathrm{C} 12 \mathrm{SH}$ thiol at the interface of the particles. However, when sonicated at a higher temperature, around $50{ }^{\circ} \mathrm{C}$, the size of the same $\mathrm{Ga}$ nanoparticles doubles ( $\sim 60 \mathrm{~nm})$ despite having additional interfacial stabilization by $\mathrm{C} 12 \mathrm{SH}$ as shown in Fig. 3e. Since temperature and surfactants serve as critical processing parameters for determining the size and surface properties of the synthesized LM nanodroplets, a dynamic temperature control (DTC) system for improving the production of LM nanoparticles via probe sonication has been recently introduced. ${ }^{69}$ Using DTC during sonication is beneficial because it enables a high concentration of LM nanoparticles to be produced by preventing the solvent from overheating and evaporating. For example, pulsed sonication of bulk LM in solvent for 15 minutes with DTC can produce large concentrations of LM NPs with an average size of $50 \mathrm{~nm}$ (Fig. 3f). The same LM nanoparticles can also be simultaneously grafted with stabilizing polyethylene glycol polymer brush (bPEG) at the interface for their long-term colloidal stability.

To produce nanoscale LM particles, longer sonication time is required but this often causes excessive production of heat in the solvent-LM mixture. Consequently, this longer processing duration can elevate the synthesis temperature and cause excessive oxidation, dealloying, and an undesirable shape or phase transformation in the generated LM nanoparticles as shown in Fig. 3g. ${ }^{70}$ Moreover, when sonicating a LM in an aqueous solvent without a surfactant, the observed phase and shape transformations of the LM nanoparticles become more prevalent. $^{26}$ This happens due to the uncontrollable production of ellipsoidal shaped gallium oxide monohydroxide (GaOOH) which causes $\mathrm{Ga}$ to be separated from its constituent elements (i.e., indium and tin) of the eutectic metal alloy. One strategy to mediate the production of $\mathrm{GaOOH}$ and make the shape of the generated LM nanoparticles controllable in water is utilizing water-soluble melanin nanoparticles (MNPs) as the additional protective layer. This is possible because MNP exhibits strong binding affinity with the Ga oxide layer while also being compatible and stable in water. ${ }^{42}$ In addition, the incorporation of MNPs allows the synthesis of various EGaIn nanostructures, ranging from nanorice, nanosphere, and nanorod. With MNPs as the protective agent, the following shapes are now regulatable by selecting the optimum amount of MNPs, sonication time, and temperature of the aqueous solvent during the synthesis of LM nanoparticles.

Other researchers have engineered an alternative experimental setup to synthesize LM nanoparticles with high controllability and repeatability over droplet sizes with their fundamental working mechanism still being reliant on sonication-based processing.
For example, a steady synthesis of EGaIn nanoparticles is possible by nebulizing liquid within an aqueous medium using a mini ultrasonication platform. ${ }^{71}$ This ultrasonication platform induces a collapse of vapor cavities to create LM nanoparticles that can be surface functionalized using various surfactants such as branched polyethylene glycol (bPEG), poly(methyl vinyl etheralt-maleic acid) (PMVEMA), and oleic acid. The benefit of this synthesis method is that the distribution and concentration of the LM droplets can be controlled by the applied DC voltage on the piezoelectric actuator of the device (Fig. 4a). For example, the concentration of the NPs can be increased from 0.5 to $5 \mathrm{mg} \mathrm{mL}^{-1}$ by increasing the input voltage from $18 \mathrm{~V}$ to $27 \mathrm{~V}$. In this ultrasonication approach, the applied voltage determines the median diameter of the generated LM particles instead of the processing duration. Hence, this methodology enables better control over the size of the EGaIn droplets than sonicationbased processes, but the synthesis requires the use of a specialized hardware. Another unconventional synthesis method combines bath sonication and a microfluidic-enabled platform to generate LM nanoparticles with tunable size distribution in an aqueous medium. ${ }^{72}$ This method injects bulk EGaIn/Galinstan

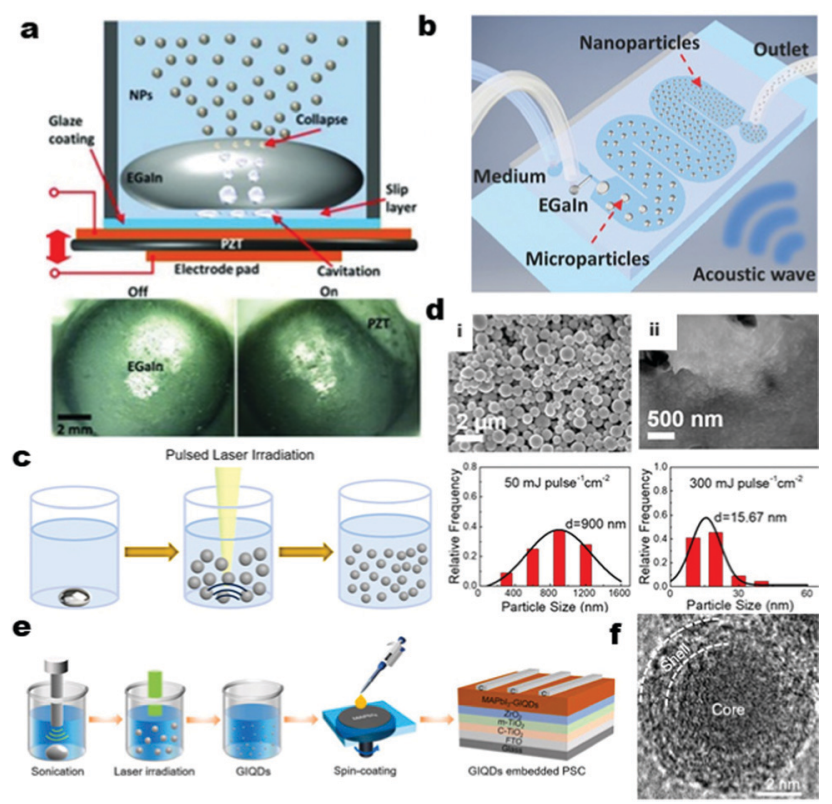

Fig. 4 (a) Production of EGaln nanoparticles using a designed mini ultrasonic platform and the mechanism for producing EGaln nanoparticles with snapshots showing the EGaln droplet within the device before (lower left) and after (lower right) activating the piezo transducer. Reproduced with permission, ${ }^{71}$ copyright 2019, Wiley-VCH. (b) Generating liquid metal micro/nanoparticles using bath sonication and a microfluidic enabled platform. Reproduced with permission, ${ }^{72}$ copyright 2018, Wiley-VCH. (c) Experimental steps for synthesizing Galinstan nanodroplets with laser irradiation and sonication and (d) synthesized Galinstan droplets reduced in size from 50 to $300 \mathrm{~mJ}$ pulse $\mathrm{cm}^{2}$ laser fluence after sonication. Reproduced with permission, ${ }^{73}$ copyright 2021, American Chemical Society. (e) Fabrication steps to create ultrasmall LM nanoparticles as gallium indium quantum dots using sonication and laser irradiation. (f) TEM image of the obtained LM nanoparticles using $150 \mathrm{~mJ}$ pulse $\mathrm{cm}^{2}$ after 10 min of sonication. Reproduced with permission, ${ }^{74}$ copyright 2021 , Elsevier. 
droplets through a serpentine patterned microfluidic channel in an aqueous medium and disrupts the LM droplets into micro/ nanosized particles with the help of ultrasonic acoustic waves as they make their way downstream (Fig. 4b). With the addition of bPEG as the surfactant to the aqueous medium, the generated LM micro/nanoparticles can also be simultaneously grafted with a stabilizing polymer brush around the oxide interface. The main benefit of this approach over probe sonication is that the dispersed LM nanoparticles are only briefly ( $\sim 15 \mathrm{~s}$ ) exposed to high ultrasonic energy inside the microchannel, which reduces the chances for EGaIn particles to dealloy and undergo a shape transformation due to prolonged exposure to high temperature in an aqueous medium. However, the reported smallest average size of LM particles generated via this approach is in the order of hundreds of nanometers which is larger than most LM particles derived using the probe-sonication method. Despite the lack of control over the polydispersity of the generated LM particles, sonication of bulk LM is one of the easiest methods to make metallic nanomaterials in comparison to the multiple processing steps and special conditions usually involved in deriving nanomaterials from solid metals.

\subsection{Sonication with laser irradiation}

Probe-based ultrasonication can also be coupled with laser irradiation to generate ultra-small $(\sim 16 \mathrm{~nm})$ core-shell structured Galinstan nanoparticles (Fig. 4c).$^{73}$ The size of these LM nanoparticles is much smaller than other reported sizes (30 to $100 \mathrm{~nm}$ average diameter) of LM nanoparticles created by using only probe sonication. ${ }^{29,55}$ After the initial step of downsizing bulk LM into the micro/nanoscale, laser irradiation further reduces the size of LM droplets by locally evaporating them into smaller, resolidified core-shell fragments. By increasing the power of laser irradiation from 50 to $300 \mathrm{~mJ} \mathrm{pulse} \mathrm{cm}^{-1}$, ultra-small Galinstan nanoparticles can be successfully generated with final average sizes of $900 \mathrm{~nm}$ and $15.67 \mathrm{~nm}$, respectively (Fig. 4d). When these nanoparticles are embedded in a commercial glue matrix, a LM-based anisotropic conductive adhesive (conductive in the thickness direction) is obtained for the interconnections of nanoelectronics. Furthermore, S. Li et $a .^{74}$ took advantage of this combined synthesis method to further reduce the size of the EGaIn nanoparticles into quantum dot particles (Fig. 4e). When these synthesized EGaIn quantum dots ( $\sim 5$ to $9 \mathrm{~nm}$ size) (Fig. $4 \mathrm{f}$ ) were spin coated as a perovskite film for solar cells, the power conversion efficiency of this solar cell improved by $\mathbf{1 7 . 1 8 \%}$ in comparison to those of solar cells without embedded EGaIn quantum dots.

\section{Embedding and self-encapsulation in polymers}

\subsection{Mechanical shear mixing}

Planetary shear mixing of two liquid phases using a dual axis centrifugal mixer is a common approach to synthesize liquid metal polymer composites. The final size of the embedded liquid droplets depends on the viscosity of each liquid phases, their volume ratio, speed, and duration of shear mixing. Initially, elastomer composites with LM inclusions with the average size varying from 20 to $140 \mu \mathrm{m}$ were synthesized using mechanical shearing without an impeller. ${ }^{35,75-77}$ In a recent effort, an overhead stirrer was used for in situ formation of EGaIn particles with an average size of $2 \mu \mathrm{m}$ for a $50 \%$ volume fraction EGaIn-PDMS composite. ${ }^{30}$ In this process, the overhead stirrer or immersion mixer, commonly used for highly viscous fluids, applies intense mechanical shear forces to the polymer and LM to achieve the desired LM droplet size. Fig. 5a shows the SEM image and 3D Nano-CT of the LM polymer composites that remain compliant and fully functional at extremely low temperatures $\left(<-80{ }^{\circ} \mathrm{C}\right)$. In a more recent study, Ford et $a .^{78}$ conducted a systematic study on the role of processing parameters (i.e., RPM and time) in the structural and functional properties of the LM composites that were synthesized using this method (Fig. 5b). For example, at 200 RPM and 1500 RPM overhead mixing for $300 \mathrm{~s}$, the obtained average radii of the suspended LM droplets are 2.8 and $0.8 \mu \mathrm{m}$, respectively. On the other hand, when the mixing RPM is fixed at 1500 RPM, the average radii of the LM droplets decreases to $5.8 \mu \mathrm{m}$ and $0.8 \mu \mathrm{m}$ after $10 \mathrm{~s}$ and $300 \mathrm{~s}$ of total mixing time, respectively (Fig. $5 \mathrm{~b}$ and c). Despite processing simplicity, the synthesized LM droplets have higher polydispersity compared to those synthesized via sonication.

\subsection{LM initiated polymerization}

3.2.1 With initiator molecules. It is inevitable that mechanical mixing and sonication of bulk LM alloys in polymer precursors or organic solvents often result in large variations in shapes and sizes of the LM micro/nanoparticles. These variations are likely caused by the formed small LM droplets

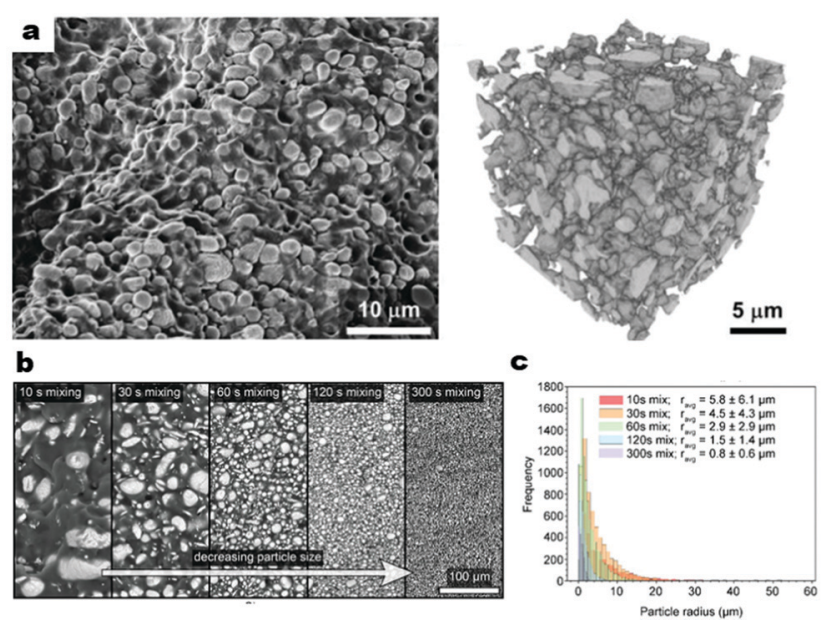

Fig. 5 (a) SEM (left) and 3D Nano-CT (right) images of the LM composite with average diameter $\sim 2 \mu \mathrm{m}$ embedded in Sylgard 184. Reproduced with permission, ${ }^{30}$ copyright 2019, Wiley- $\mathrm{VCH}$. (b) Microscopy images of crosssections of LM composites with Sylgard 184 as the matrix to show the influence of mixing time on the size of the final droplets. Mixing rate was fixed at 1500 RPM; (c) the average LM particle radius as a function of mixing time for fixed 1500 RPM. Reproduced with permission, ${ }^{78}$ copyright 2020, Wiley- $\mathrm{VCH}$. 
recoalescing during and after the synthesis because of the deformable and chemically sensitive oxide shell. Several studies have already demonstrated that the thin passivating oxide layer (without chemical modification) has a lower mechanical strength, ${ }^{55,56,79}$ is etchable in high or low $\mathrm{pH}$ environments, ${ }^{31,80,81}$ and can be weakened in water. ${ }^{82}$ To ensure that these LM nanoparticles are suitable to produce highly isotropic, homogeneous, and functional (stable elastic, thermal, and dielectric properties) nanocomposites, a uniform sphere shape size distribution and a high-volume fraction of suspended LM nanoparticles are desirable. An approach to fulfill these criteria is to form polymer coatings on LM nanoparticles that are compatible with the encapsulating polymer in the nanocomposite. ${ }^{83}$ These polymer-coated LM nanoparticles can be synthesized by initiating in situ radical polymerization at the oxide interface.

To execute in situ polymerization at the oxide interface of LM nanoparticles, a surface-initiated atom transfer radical polymerization (SI-ATRP) reaction is facilitated through probebased ultrasonication. ${ }^{83}$ Generally, an SI-ATRP reaction is a process that begins by first attaching polymer initiators (i.e., cross-linkers) on the Ga oxide layer followed by exposure to the corresponding monomers. A follow-up study has shown that in situ polymerization by SI-ATRP on EGaIn nanodroplets can occur without sonication assistance but the following reaction is found to be several orders of magnitude slower. ${ }^{84}$ In the first study on synthesis of EGaIn nanodroplets through SI-ATRP, the particles were synthesized using both sequential sonication (Fig. 6a) or a one-pot sonication (Fig. 6b). For example, to form EGaIn nanoparticles with poly(n-butyl methacrylate) (PBMA) coating, EGaIn droplets are first tethered with 12-(2-bromoisobutyramido)dodecanoic acid (BiBADA) in tetrahydrofuran (THF) prior to the SI-ATRP reaction with $n$-butyl methacrylate monomers. For one-pot synthesis, a single sonication step is done without THF. The generated nanosized PBMAcoated EGaIn nanodroplets (Fig. 6c) can be embedded in a pure PBMA matrix up to $40 \%$ volume fractions with the resulting LM nanocomposite exhibiting high stretchability (Fig. 6d) even at low temperatures because of the supercooling properties of the embedded LM nanoparticles. Another study facilitated SI-ATRP reaction by using oleic acid as an alternative biofriendly initiating
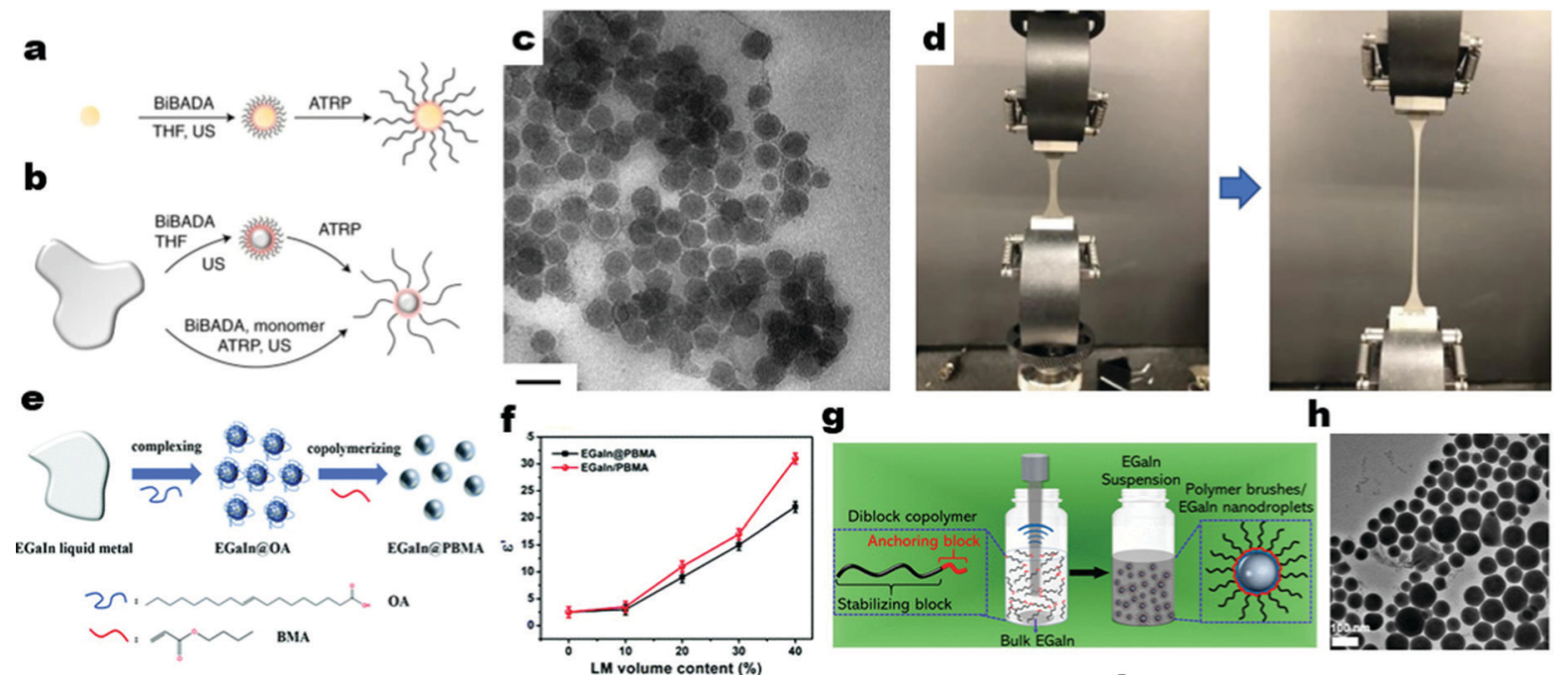

f

g

h
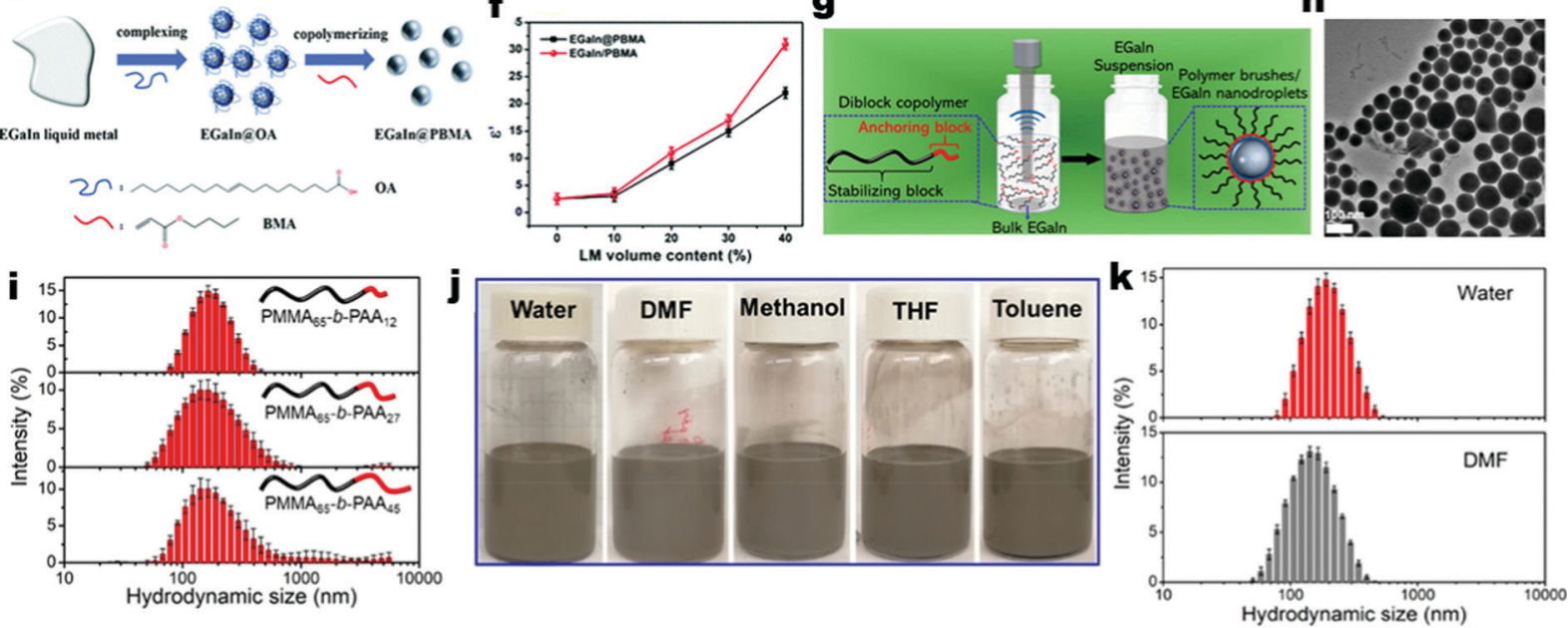

Fig. 6 (a) Two-step ultrasonication with BiBADA functionalization and SI-ATRP of nanoparticles, (b) one-step ultrasonication with BiBADA monomer and EGaln to induce SI-ATRP, (c) transmission electron microscopy (TEM) image of the supernatant of the EGaln-PBMA dispersion in THF. Scale bar, $200 \mathrm{~nm}$, (d) tensile test of a PMMA-b-PBA-grafted EGaln hybrid material specimen. Reproduced with permission, ${ }^{83}$ copyright 2019, Nature. (e) The mechanism of synthesizing core-shell structured EGaln-PBMA nanodroplets using oleic acid (OA) as the initiator, (f) frequency-dependent dielectric constant $\left(\varepsilon^{\prime}\right)$ of EGaln-PBMA nanocomposites with different LM volume loadings. Reproduced with permission, ${ }^{85}$ copyright 2020 , Royal Society of Chemistry. (g) Illustration of the design of attachable diblock copolymer and fabrication of polymer brushes/EGaln nanodroplets by ultrasonication, (h) TEM image of the PMMA-b-PAA/EGaln nanodroplets, (i) effect of DP of the PAA block on the average size and size distribution of PMMA- $b$-PAA/EGaln nanodroplets, (j) images of suspensions of nanodroplets prepared in different solvents, $(k)$ the resultant size distribution of LM nanodroplets in water and DMF. Reproduced with permission, ${ }^{86}$ copyright 2020, American Chemical Society. 
agent for assembling PBMA monolayers on EGaIn nanoparticles (Fig. 6e). ${ }^{85}$ These nanoparticles, with an average diameter of $\sim 300 \mathrm{~nm}$, can create LM nanocomposites with $\sim 400 \%$ stretchability (i.e., comparable to pure PBMA) while showing a highly effective dielectric constant in the range of 30 (Fig. 6f). These improvements in the functional properties can be attributed to the smaller and narrower size distribution enabled by the polymer-coated LM nanoparticles.

Adding surface-tethered macromolecules or polymer brushes on EGaIn nanodroplets, such as diblock copolymers with short poly(acrylic) acid (PAA) segments, rather than tethered initiators for surface polymerization can also enable the formation of a protective interface. ${ }^{86}$ With this additional coating, a high yield of narrow size distributed EGaIn nanoparticles can be synthesized. The diblock copolymers consist of attachable poly(methyl methacrylate)-block-poly(acrylic acid) (PMMA- $b$-PAA) where the PAA chains act as the anchoring block onto the oxide layer whereas the longer PMMA chain plays the role of a stabilizer, preventing LM nanodroplets from coalescing (Fig. 6g). To conduct this synthesis for creating the smallest LM nanodroplets (Fig. 6h), bulk EGaIn is ultrasonicated with PMMA- $b$-PAA copolymer in dimethylformamide (DMF) solution. By increasing the length of PMAA (stabilizer) polymer chain within the PMAA- $b$-PAA copolymer, the size distribution of the yielded LM nanoparticles has smaller standard deviation but it becomes more polydisperse (Fig. 6i). For stabilizing EGaIn nanodroplets in both water and organic solvents (Fig. 6j), poly(ethylene glycol)methyl ether-blockpoly(acrylic acid) (PEO- $b$-PAA) is another diblock copolymer with a water-soluble polymer block (PEO) that can be attached to the oxide interface. The results show that the size distribution of the LM nanodroplets in DMF and water is similar when this water-soluble diblock polymer is attached at the oxide interface (Fig. 6k). Moreover, the successful attachment of polymer brushes on the oxide skin also enables the possibility of attaching polymer brushes that are compatible with photolithography. ${ }^{87}$ Thus, with selective photolithographic patterning, spatial and temporal control of surface polymerization at the surface of LM nanoparticles is now possible.

Creating a conductive polymer layer on LM nanoparticles is also possible by in situ polymerization. One novel method is to grow a conductive polyaniline nanofibrous network directly on the insulative $\mathrm{Ga}$ oxide interface of EGaIn nanoparticles. ${ }^{88}$ Growing the polymer from the surface of LM nanoparticles by in situ polymerization is beneficial because the functional groups of the conductive polymers are susceptible to irreversible degradation when deposited or exposed to metal ions such as $\mathrm{Ga}^{3+}$. To enable in situ polymerization, the reactive LM droplet is first disrupted by sonication to expose the reactive LM with $p$-phenylenediamine followed by aniline monomers and their initiator (ammonium peroxydisulfate). The created polyaniline layered LM nanoparticles have been applied as sensitive sensors for detecting acidic vapor and temperature changes. Recently, another strategy to create conductive interfaces on LM micro/ nanoparticles is demonstrated by hydrogen doping of the oxide layer. ${ }^{89}$ This conductive hydrogenated oxide skin is spontaneously formed when sonicating bulk EGaIn/Galinstan with poly(ethylene-co-vinylacetate) and a free radical initiator (dicumyl peroxide). Once prepared as an ink to print conductive traces, the LM particles require no sintering as interparticle contact is sufficient to form electrical connections. As a result, the printed conductive circuit using this hydrogen doped LM particles is robust against scratching, temperature, and humidity changes and exhibits no resistance change even after $500 \%$ of uniaxial strain.

3.2.2 Without initiator molecules. So far, the reviewed LM initiated polymerization processing methods employed molecular initiators for grafting polymers around LMNPs. However, LM itself can initiate surface polymerization during sonication. This is evident when EGaIn is present in an aqueous solution of vinyl monomers, a rapid free radical polymerization (FRP) can occur without requiring the use of molecular initiators. ${ }^{90}$ In this process, the high energy ultrasonication is used to not only reduce the size of bulk LM but also simultaneously expose the reactive Ga alloy core to directly initiate polymerization by FRP with an available vinyl-based monomer such as acrylamide (Fig. 7a). This process eventually results in the formation of a thick hydrogel layer (20 nm thick polyacrylamide - PAAm) on the interface of LMNPs which appears as a bulk gel after sonication (Fig. 7b). When these hydrogel grafted LM nanoparticles are embedded in liquid metal PAAm nanocomposites, the composite exhibits both thermal responsiveness (Fig. 7c) and high stretchability up to $1500 \%$ strain (Fig. 7d). Ring opening polymerization is another surface polymerization approach on LM particles without requiring initiator molecules. ${ }^{91}$ This polymerization technique is initiated when sonicating bulk EGaIn with fluidic lactones. Through this method, EGaIn nanodroplets grafted with polylactone shells are produced. The thickness of the polylactone shell is tunable and transforms LMNPs into a stable and durable solid powder that can be dispersed in different organic solvents for synthesis of various liquid metal polymer nanocomposites. Based on these studies, it is evident that liquid metal nanoparticles generated via sonication can initiate and catalyze surface radical polymerization without requiring initiators. Therefore, other studies have taken advantage of the catalytic properties of surface oxides to effectively convert various water-soluble monomers into long-chained high molecular weight polymers with or without the help of sonication. ${ }^{84,92}$

Another method to decorate LM with different types of polymers is the reversible addition-fragmentation chain transfer (RAFT) process. ${ }^{93}$ In this process, a RAFT agent is first anchored on the generated EGaIn nanoparticles during ultrasound treatment in THF before being exposed to monomers to initiate surface polymerization (Fig. 7e). The RAFT agent in this study has a carboxylic group (HOOC) for binding to the Ga oxide layer, long alkyl chain for stabilization, and a trithiocarbonate group for enabling polymer chain growth when monomers are present. The study shows that with the proper design and choice of RAFT agents tethered on the interface of LMNPs, a diverse group of polymers and block copolymers can be grafted at the interface to allow EGaIn nanoparticles to have better dispersion stability. For instance, when methyl 
a

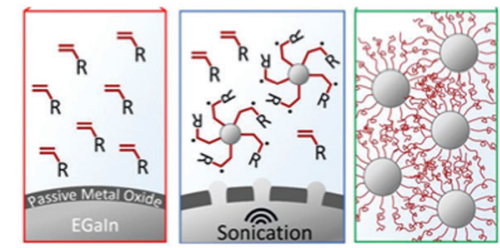

e
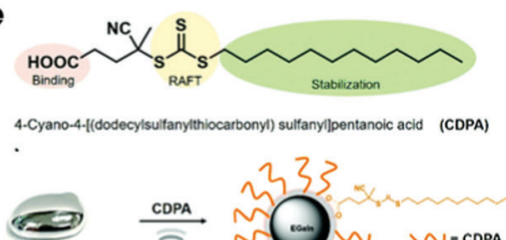

EGain

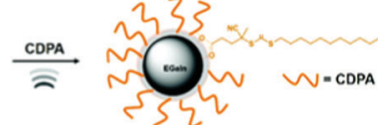

b
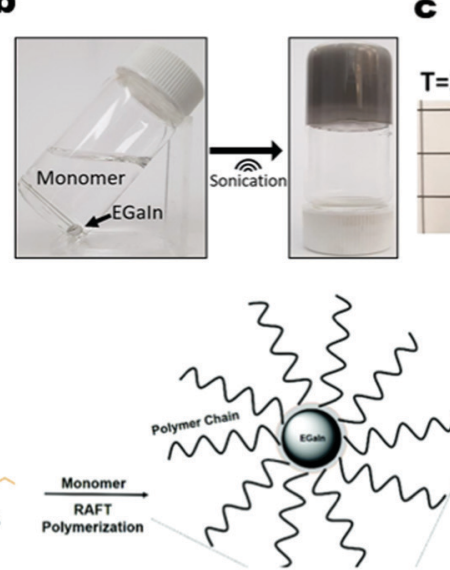

$c$

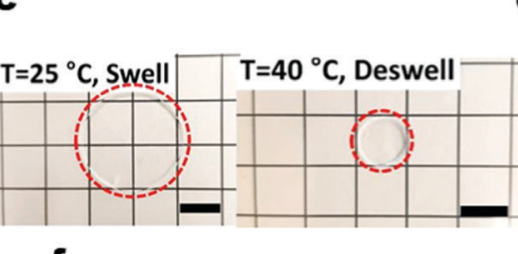

f

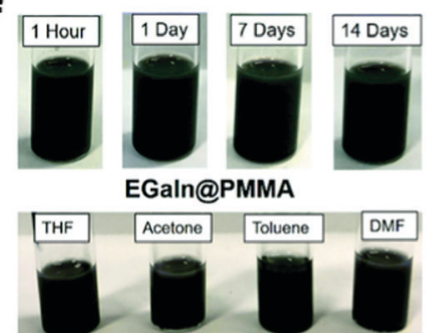

d $\lambda=15$

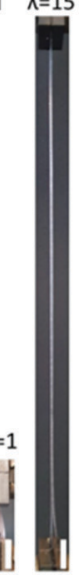

Fig. 7 (a) A passivating oxide layer isolates the metal from the solution. Sonication breaks the liquid metal into small particles and thereby greatly increases the surface area and exposes the Ga core, which initiates radical polymerization, (b) LM particles initiate free radical polymerization without the need for conventional molecular free-radical initiators, (c) swelling and deswelling of the PAAm LM composite when responding to lower and higher temperatures. Scale bars correspond to $10 \mathrm{~mm}$. (d) PAAm LM composite showing stretchability ( $\lambda$ ) of $1500 \%$. Reproduced with permission, ${ }^{90}$ copyright 2019, American Chemical Society. (e) Top: The structure of the RAFT agent. Below: Processing schematic during sonication and the polymer chain assembly on LMNPs, (f) the stability test of EGaln with the PMMA layer over 14 days in THF and the dispersibility test in different solvents. Reproduced with permission, ${ }^{93}$ copyright 2021, Royal Society of Chemistry.

methacrylate (MMA) monomers are added, PMMA is successfully grafted around EGaIn nanoparticles to provide long-term stability in various solvents (Fig. 7f).

A challenge that remains for most surface initiated ATRP and RAFT-based synthesis of LM nanoparticles is that they are limited to low monomer concentrations which results in a low yield of surface polymerization. This is because at a high concentration of monomers, the large amount of polymerization results in increased viscosity of the solvent-LM mixture which hinders the strength of cavitation bubble induced by ultrasonication. Moreover, these processes are not readily scalable and require additional studies on the scalability and stability of the final nanomaterials when synthesized in larger quantities.

\section{Surface modification}

For liquid metal nanodroplets to be compatible with various advanced additive manufacturing techniques such as inkjet, aerosol printing, and direct writing, a stable oxide interface that will grant long-term dispersions and particle-solvent compatibility is highly desirable. An approach to achieve these functionalities is to specifically engineer the oxide interface of the LM droplets for creating a surface versatile and printable LM nanoparticle ink. Other than this, surface modification of LM nanoparticles is vital for designing multifunctional polymer nanocomposites, stretchable conductors, and biocompatible therapeutic nanocarriers with tailorable properties. In the previous section, the discussed surface modification methods involve creating a surface polymer layer at the interface of LM droplets through a "growing from" approach. An alternative approach will be to deposit or chemically modify the Ga oxide interface with various inorganic and organic materials.

\subsection{Functionalized LM nanoparticles with inorganic and organic coating}

Inorganic agents can control the thickness and stiffness of the native oxide skin of LM nanoparticles for a functional purpose. For instance, the addition of highly oxidizing hydrogen peroxide in ethanolic solution for probe sonication of bulk EGaIn can produce EGaIn droplets with a thick oxide shell, $\sim 4 \mathrm{~nm}^{56}$ Also, these synthesized LMNPs with thicker $(\sim 4 \mathrm{~nm})$ oxide shells have larger measured elastic modulus ( $>2 \mathrm{GPa}$ ) than those of unfunctionalized LM droplets ( $\sim 1.06 \mathrm{GPa})$ (Fig. $8 \mathrm{a}$ ) and thus are more challenging to sinter on a nanoscale due to the rigid insulating oxide shell. ${ }^{55}$ On the other hand, surface chemical functionalization with various thiolated ligands can suppress the Ga oxide growth at the interface of the LM droplets by reducing the available surface sites for oxygen adsorption (Fig. 8b). ${ }^{11}$ For example, thiolated ligands such as dodecanethiol (DDT) and 2,3,4,5,6-pentafluorothiophenol (FTP) suppress the thickness of the oxide shell by $30 \%$ and $23 \%$, respectively, 1 day after synthesis (Fig. 8c). After allowing 30 days for both the functionalized and unfunctionalized LM nanodroplets to mature, the DDT and FTP functionalized LM nanodroplets were still found to have measured oxide shell thicknesses that are $13 \%$ and $17 \%$ smaller than those of unfunctionalized LM droplets $(\sim 2.6 \mathrm{~nm})$. From this study, it is known that FTP is the best thiol ligand for slowing the final growth of the oxide shell but the decline in thiol-metal (i.e., Ga core) bonding over time still leads to inevitable regrowth of the oxide shell on LM nanodroplets (Fig. 8c).

To ensure that the Ga oxide growth on LM nanodroplets is irreversible while still having a tunable thickness, 1-Decylphosphonic acid (DPA) has been subsequently chosen as an alternative ligand molecule for the synthesis of LM droplets. ${ }^{95}$ The benefit of the DPA ligand is that it can form strong and 

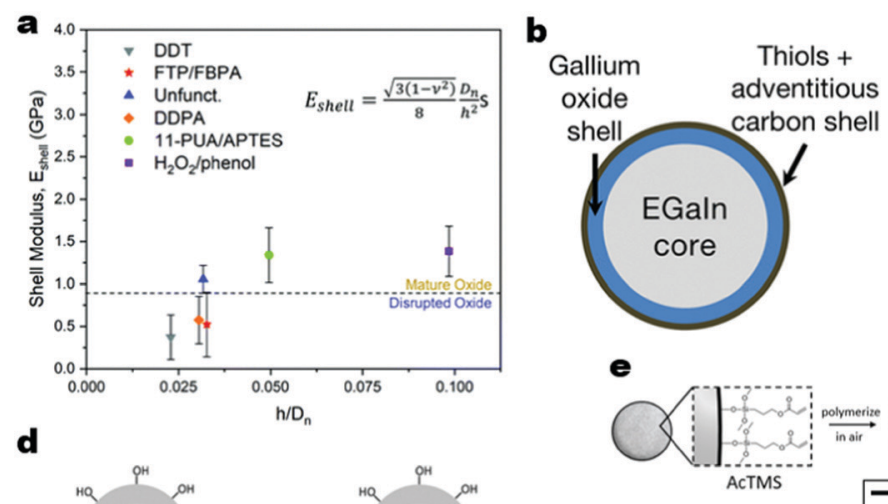

C

d
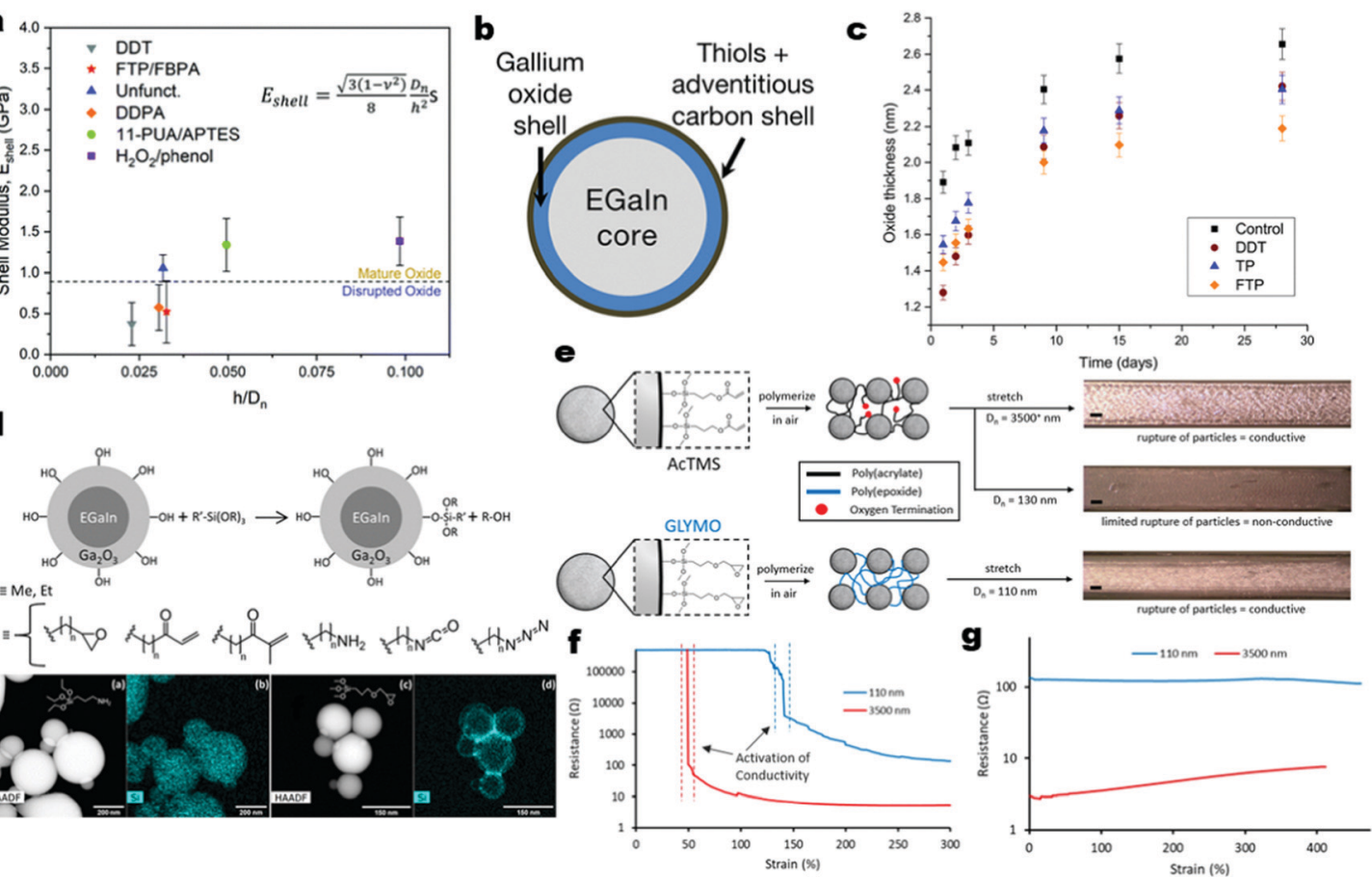

Fig. 8 (a) Ga oxide shell elastic modulus against particle thickness to diameter ratio for EGaln nanoparticles with various chemical functionalization methods. Reproduced with permission, ${ }^{56}$ copyright 2019, Royal Society of Chemistry. (b) The core-shell structure of a LM nanoparticle; it is likely that the gallium oxide shell is not totally continuous in thiolated nanoparticles as thiols are expected to bind to elemental gallium rather than gallium oxide, (c) initially, oxide thickness is reduced up to $30 \%$ by thiolation, while at the longest times studied thiolation produces up to a $17 \%$ thickness reduction. Although DDT starts out with a thinner oxide shell initially, as equilibrium is approached, the FTP sample exhibits a thinner oxide than either TP or DDT. Reproduced with permission, ${ }_{11}^{11}$ copyright 2018, American Chemical Society. (d) Silanization of the EGaln particles with schematic depicting the reaction of EGaln particles possessing hydroxylated gallium oxide shells with substituted trialkoxysilane, (e) polymerized LM network formation with straininduced rupture at much smaller particle sizes than the oxygen-inhibited free-radical polymerization of acrylates, electromechanical behavior of functionalized polymerized LM networks for initial strain activation (f) and strain to substrate failure (g). Reproduced with permission, ${ }^{94}$ copyright 2020 , American Chemical Society.

long-term covalent bonds on the Ga oxide interface as proven before on bulk EGaIn surfaces. ${ }^{96}$ Moreover, the binding strength of the DPA layer on the Ga oxide surface improves even after being stored for a week under ambient conditions or can be further augmented by heat treatment (i.e., heat reflux in solvent or bath sonication). In contrast, the thiol-Ga binding strength is short-lived which allows less stable Ga oxide shell to regrow on LM nanodroplets.

Another way to circumvent the issue of transient surface functionalization among thiol ligands is to instead silanize liquid gallium alloy nanoparticles. The silanization of LM nanoparticles can be performed by enabling the reaction of hydroxyl species on the oxide layer with the alkoxysilane group $\left(\mathrm{R}^{\prime}-\mathrm{Si}(\mathrm{OR})_{3}\right)$ of a substituted trialkoxysilane $\left(\mathrm{R}^{\prime}\right)$ such as 3-(glycidyloxypropyl)-trimethoxysilane (GLYMO) and 3-(acryloxypropyl)-trimethoxysilane (AcTMS) as illustrated in Fig. 8d. ${ }^{94}$ The following chemical functionalization on the interface enables diverse chemical groups (i.e., amines, epoxides, cyanides, nitriles, amides, and esters) including the more expensive DPA ligand to be permanently functionalized on the core-shell structure of Ga-based LM alloys. Furthermore, the alkoxysilane functionalization of LM droplets allows for the fabrication of architected polymerized liquid-metal networks, termed poly-LMNs, with tunable mechanoresponsive stretchable conductors (Fig. 8e). These poly-LMNs with nanosized $(\sim 110 \mathrm{~nm})$ GLYMO functionalized LM droplets can be conductive at a small strain although the rigid insulative oxide shell of the LM nanoparticles can be difficult to rupture and there is significantly less volume of conductive LM (EGaIn core) available compared to insulating Ga oxides at the nanoscale (Fig. 8f). For instance, poly-LMNs with LM nanoparticles functionalized with GLYMO exhibit successful strain activation (i.e., from dielectric to conductor) at $150 \%$ strain and have negligible electromechanical behavior up to $400 \%$ strain. In contrast, poly-LMNs with nanosized $(\sim 130 \mathrm{~nm})$ AcTMS functionalized LM droplets show limited rupturing to form a conductive network at the same strain (Fig. 8g). This distinction occurs because the GLYMO surface functionalization enables a higher order of interparticle polymerization. As a result, the LM nanoparticles with GLYMO functionalization can be easily sintered to form percolated conductive networks after straining.

\subsection{Functionalized LM nanoparticles for biocompatibility and stability in water}

Most surface modifying and functionalizing molecules, including thiolated ligands, involve the stabilization of suspended LM micro-/nanoparticles in toxic organic solvents (i.e., ethanol, 
hexane, and benzene). However, for biomedical applications, where bulk or nanodroplets of LM have the potential to carry and release therapeutic payloads in the human body, an alternative non-toxic protective layer surrounding the LM particle in an aqueous medium is essential. The challenge of synthesizing LM droplets by ultrasonication in water (an oxygen-rich environment) is that the pristine Ga core can undergo rapid uncontrollable oxidation to form undesirable gallium oxide monohydroxide $(\mathrm{GaOOH})$ solid nanorods, particularly as the water temperature increases. ${ }^{97}$ Simultaneously, indium particles covered with $\mathrm{GaOOH}$ spiky rods can also form when ultrasonicating bulk EGaIn in a water-ethanol mixture, indicating the dealloying of the LM alloy (Fig. 9a). ${ }^{94}$ Although these formed $\mathrm{GaOOH}$ crystallites can be stable in water and silanized, and have efficient photothermal transduction, they lack the desired core-shell structures for other functional applications.

A protective barrier such as a water-soluble polymer can be deposited on the core-shell structure of LM nanoparticles to avoid uncontrollable oxidation. Hence, coating liquid metal nanoparticles with water-soluble polymers such as poly(vinylpyrrolidone) (PVP) is a rapid method to create stable core-shell LM particles in an aqueous medium. ${ }^{98}$ This is achieved by degrading PVP into smaller fragments by ultrasonic cavitation bubbles before directly depositing onto EGaIn as a coating shell with a thickness of up to $20 \mathrm{~nm}$ (Fig. 9b). For comparison, this PVP coating shell is much larger than the thiol ligand-derived protective shell $(<5 \mathrm{~nm})$. As a result, the PVP-coated LM coreshell particles have longer stability of 30 and 60 days in water and ethanol, respectively, while it has been reported that thiol-coated LM particles have $<7$ days of colloidal stability. ${ }^{25}$ In addition, with PVP coating in an aqueous medium, the generated LM nanoparticles are both smaller and less polydisperse (compared to without PVP) (Fig. 9c). This is different than LM nanoparticles with a polymer brush (diblock copolymers) coating where the obtained particle size and polydispersity are inversely proportional. Other than this, the PVP polymer deposition on LM nanoparticles is a one-step synthesis process in water (green solvent) while previous strategies to form a polymer shell around LM particles such as surface-initiated atom transfer radical polymerization and sonication-assisted emulsification involve multiple additional steps (i.e., initiation, polymerization, heat treatment, and washing steps) in addition to using different toxic organic solvents. ${ }^{32,83}$ It is discussed that when producing LM nanoparticles in water, these generated colloidal particles suffer short-term stability and have wide size distributions. To remedy both issues, EGaIn can be sonicated with poly(1-octadecene-altmaleic anhydride) (POMA) in water to produce stable, doublelayered POMA encapsulated LM nanoparticles. ${ }^{32}$ The doublelayered POMA encapsulation offers a hydrophobic insulation layer and a non-chemically reactive barrier for the dispersed LM nanoparticles because of the formed octadecyl $\left(\mathrm{CH}_{2}\left(\mathrm{CH}_{2}\right)_{x} \mathrm{CH}_{3}\right)$ hydrocarbon chain (Fig. 9d). As a result, the mean size of produced NPs in water after sonication can be as small as $170 \mathrm{~nm}$ with both high concentration and narrow size distribution.

For tunable shape and surface properties of LMNPs in an aqueous medium, a novel composite material that embeds the LM core in a zeolitic imidazolate framework (ZIF) shell is introduced. ${ }^{99}$ This is done by first stabilizing the EGaIn nanodroplets with poly(vinyl pyrrolidone) (PVP) coating during sonication (Fig. 9e). When these PVP-coated EGaIn nanodroplets are mixed with 2-methylimidazole (2-MI) and zinc ion $\left(\mathrm{Zn}^{2+}\right)$ in methanol, the coating will interact with the zeolitic

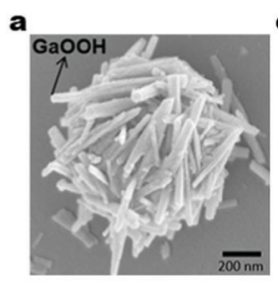

b

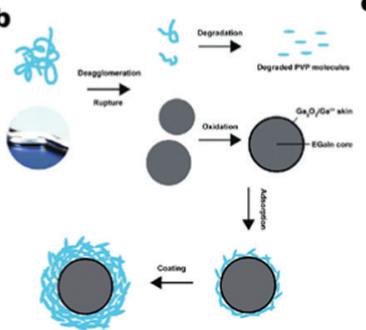

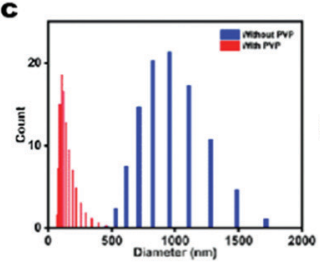

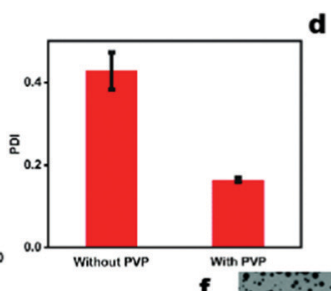

d
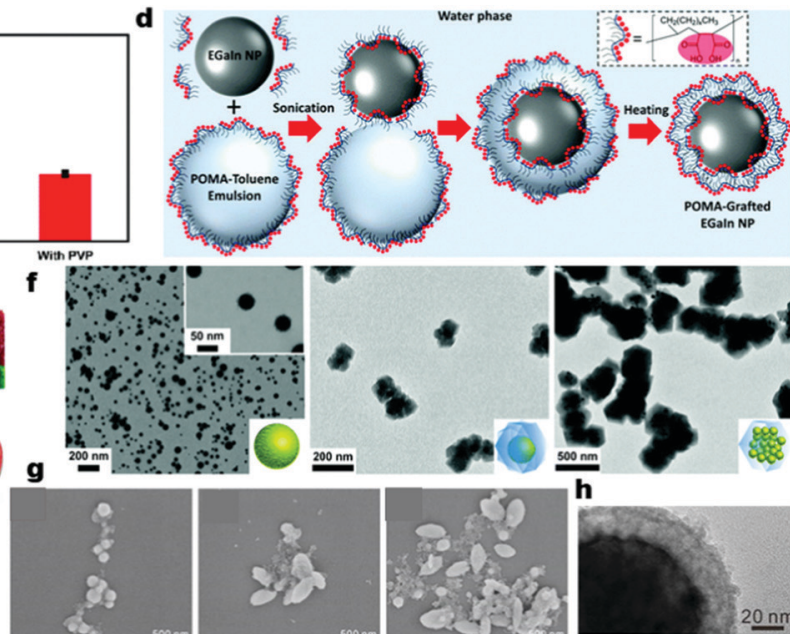
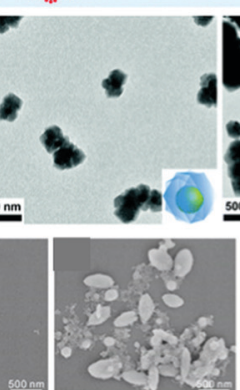

Fig. 9 (a) Crystalline spikes of the EGaln particles sonicated in the presence of water. Reproduced with permission, ${ }^{94}$ copyright 2017 , Royal Society of Chemistry. (b) PVP-assisted synthesis of LM nanoparticles in water. The degraded PVP molecules adsorb onto the oxidized surface of the EGaln nanoparticles and induce the deposition of a polymer coating, (c) size distribution and polydispersity index (PDI) of LMNPs with or without PVP. Reproduced with permission, ${ }^{98}$ copyright 2020, Wiley-VCH. (d) Production of POMA grafted EGaln NPs with schematic depicting the mechanism leading to the production of stable liquid metal NPs. Reproduced with permission, ${ }^{32}$ copyright 2018, Wiley-VCH. (e) Illustration of the synthesized "litchi"- and "passion fruit"-like EGaln with ZIF-8 nanoparticles. (f) Images of EGaln with PVP coatings (left), single (middle) or more (right) ZIF-8 crystals encapsulation. Reproduced with permission, ${ }^{99}$ copyright 2020, Royal Society of Chemistry. (g) Light-induced shape transformation of PDA coated core-shell LM nanoparticles and the (h) TEM image of a single particle. Reproduced with permission, ${ }^{100}$ copyright 2019 , Wiley-VCH. 
imidazolate framework ZIF-8 precursor for the next encapsulation stage. By changing the ratio of ligand (2-MI) to $\mathrm{Zn}^{2+}$ metal ions, the number of EGaIn nanodroplets encapsulated in single (Fig. 9f middle) or more (Fig. 9f right) ZIF-8 crystals can be controlled. Other efforts have focused on stable dispersion of core-shell LM droplets in an aqueous medium with a reversible spherical to ellipsoid shape change when stimulated by nearinfrared radiation (NIR) (Fig. 9g). ${ }^{100}$ This feature can be achieved by assembling a thick $(20 \mathrm{~nm})$ polydopamine (PDA) monolayer which has a strong photothermal conversion (i.e., light to heat energy) property around the outer oxide shell of LM nanodroplets (Fig. 9h). With this shape transformation functionality, the following PDA layered LM nanodroplets can be used in cancer treatments by controllably releasing therapeutics at the treatment site using light irradiation.

Conductive 2D inorganic materials such as graphene can also be deposited on the generated LM nanoparticles to enable stable colloidal dispersions in an aqueous medium and in a broad range of $\mathrm{pH}$ conditions. ${ }^{101}$ To synthesize such nanomaterial architecture, bulk EGaIn is sonicated in water with adjustable $\mathrm{pH}$ and pre-dispersed graphene oxides (GO). During this process, 2D graphene oxide can form a secondary protective encapsulation for EGaIn nanoparticles under acidic conditions. On the other hand, under basic conditions, only thiolated graphene oxide can be deposited on the oxide layer of the EGaIn nanoparticles. As a result, the EGaIn nanoparticles with GO encapsulation have $10 \times$ larger measured stiffness for a single nanoparticle and require $5 \times$ more compressive loading to be sintered as thin film in comparison to using pristine EGaIn nanoparticles. In short, GO encapsulation not only stabilizes EGaIn nanoparticles under various $\mathrm{pH}$ conditions but also enables their interface to be electrically conductive with regulatable rigidity.

\section{Applications of LM nanoparticles}

The applications of functional liquid metal nanoparticles have been continuously increasing in the creation of flexible and stretchable electronics as they can be made compatible with different additive manufacturing techniques (i.e., handwriting, direct writing, and inkjet printing). Nanoscale LM droplets are also utilized for creating thermal, dielectric, and solar energy harvesting materials in the form of nanocomposites. In the field of nanomedicine, nanoscale LM droplets are preferably used as therapeutic carriers or disease diagnostic tools because of their known non-toxic properties. These emerging applications of LMNPs are briefly discussed here.

\subsection{Printed stretchable conductors}

Liquid metal nanoparticles are preferrable for creating printable electronics because of the reduced surface tensions and large surface area of the chemically modifiable oxide layer. These are the two features that are crucial for the stable dispersion of LM nanodroplets as printing ink and surface adherence on various stretchable and flexible substrates. In soft electronics, bulk LM is commonly used as a microfluidic-based LM interconnection to fill the created channel, but this fabrication step requires large dispensing pressure because of the high surface tension and viscosity of bulk EGaIn. ${ }^{102,103}$ To overcome this, concentrated EGaIn microparticles in ethanol solution (formed via ultrasonication) are selected because their lower viscosity and surface tension (due to reduced droplet size) will enable a lower injection pressure into the PDMS microchannels. ${ }^{68}$ Once most of the solvent evaporates, the sandwiched EGaIn microparticles can now be selectively activated from the top of the PDMS packaging where the length of sintered conductive trace controls the resonant frequency of a soft antenna (Fig. 10a). ${ }^{68}$

Creating soft electronics by direct writing of bulk EGaIn interconnections often poses difficulties such as high surface tension and limited substrate wettability (large contact angle $\left.>90^{\circ}\right) .{ }^{104}$ Hence, inkjet printing of bulk LM is not possible. As a result, this leads to the preference towards using LM nanoparticles which exhibit lower surface tension to enable both successful nozzle and inkjet printing. ${ }^{51}$ For example, EGaIn nanoparticles (180 to $600 \mathrm{~nm}$ ) can be inkjet printed directly onto an elastomer glove to form multiple scalable arrays of strain gauges (Fig. 10b). When these LM nanoparticles are prepared as a thin film, they can also be selectively sintered using laser irradiation to form thin conductive traces of any architecture before encapsulation in protective PDMS as a soft circuit. ${ }^{60}$ This created a soft circuit that not only adheres well to the skin but is also compliant to large wrist movements (Fig. 10c). Moreover, the resistance changes of the created soft circuits are negligible even after repeated bending deformations up to 1000 cycles and, thus, perform much better than bulk LM traces (microfluidic or printed) which often exhibit larger resistance change under deformation.

Surface modification enables LM nanoparticles to maintain average size, long-term dispersion, and surface wettability or adherence with broad types of substrates. In early attempts, thiol ligated LM nanoparticles $(\sim 110 \mathrm{~nm})$ were used to produce printing ink with high dispersion stability to create high resolution printed electronics. With these LMNPs, a flexible micro-coil can be created using inkjet printing and laser patterning techniques. ${ }^{29}$ The following printed micro coil showcases stable and low resistance behavior after 100 cycles of loading (Fig. 10d). However, the bare oxide layer and other thiol ligands on the LM nanodroplets still have limited wettability with certain substrates. A recent study addresses this limitation by functionalizing natural polyphenols such as tannic acid (TA) on the interface of EGaIn to enable better adhesion of the nanoparticles with diverse rigid and soft surfaces. ${ }^{54}$ This improvement is attributed to TA molecules having universal surface adhesion properties and possessing the functional groups to anchor strongly on Ga-based LMNPs. Previously, other polyphenol compounds, such as gallic acid, have been self-assembled on Ga nanoparticles via sonication for stabilization in water and incorporation as conductive paper membranes. ${ }^{105}$ In contrast, the assembled TA molecules on EGaIn nanoparticles serve as versatile adhesives when LM nanoparticles are deposited on different types of surfaces. For functionalization, probe-based ultrasonication of bulk EGaIn in the presence of 

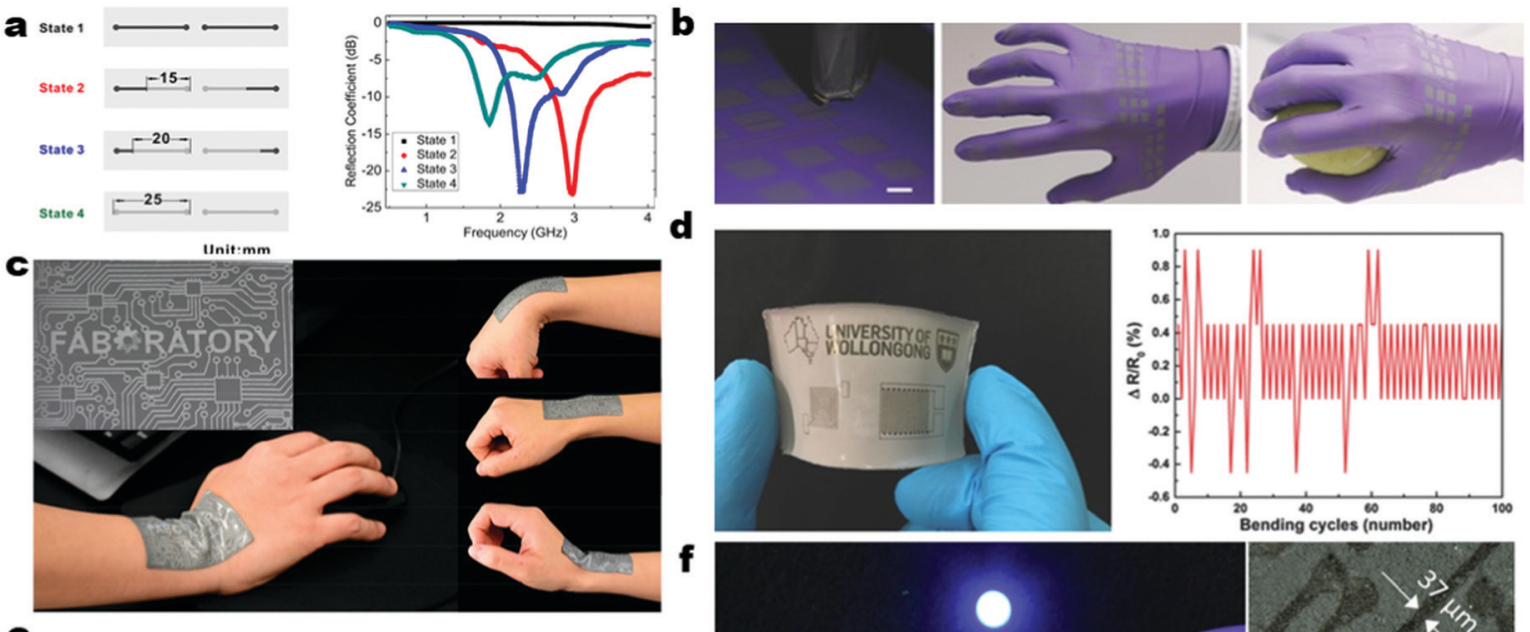

e
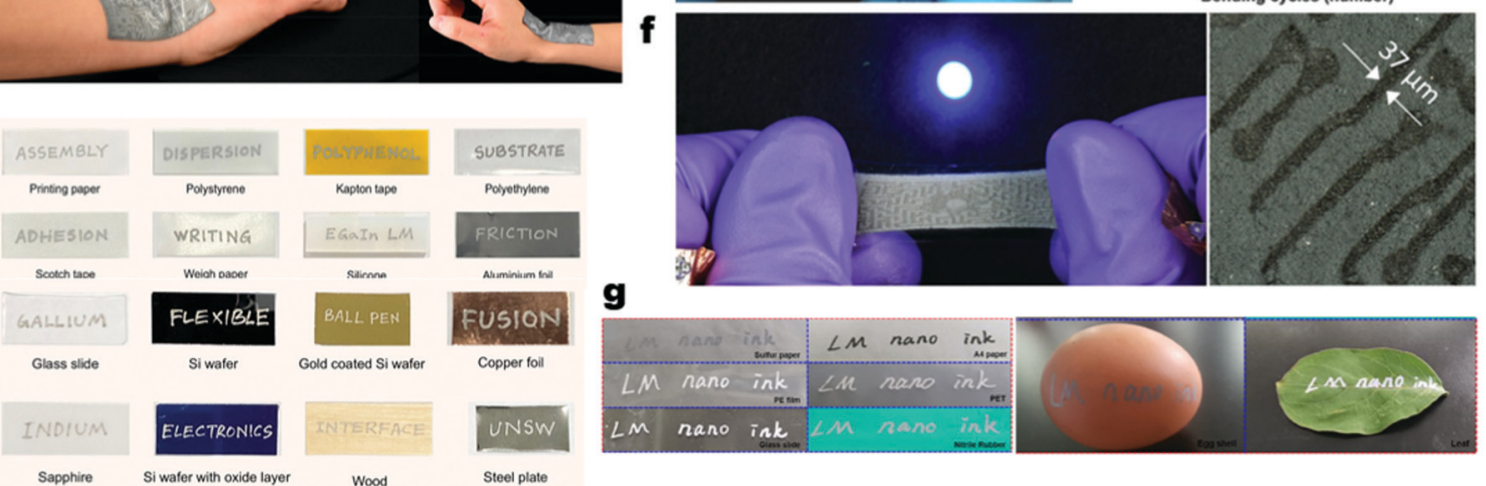

g

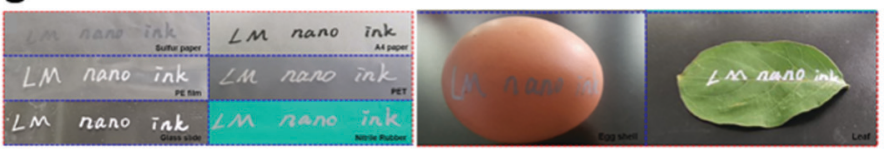

Fig. 10 (a) Different lengths of flexible antenna by selective mechanical sintering of encapsulated LM trace for tuning the resonant frequencies. Reproduced with permission, ${ }^{68}$ copyright 2015, Wiley-VCH. (b) Inkjet printing of EGaln NPs to form strain gauges on a nitrile glove. Scale bar is 5 mm. Reproduced with permission, ${ }^{51}$ copyright 2015, Wiley-VCH. (c) Soft circuit board with conductive traces made from a laser sintered EGaln nanoparticle substrate adhered to human wrist. Reproduced with permission, ${ }^{60}$ copyright 2018. American Chemical Society. (d) A flexible micro-coil electrode prepared by laser patterning from EGaln nanodroplets and the resistance stability over a hundred rounds of bending and folding (right). Reproduced with permission, ${ }^{29}$ copyright 2016, American Chemical Society. (e) EGaln-TA nanoparticle ink handwritten with ball point pen on different rigid, flexible and curved surfaces. Reproduced with permission, ${ }^{54}$ copyright 2021, Wiley-VCH. (f) Utility of the double emulsion ink to create stretchable electronics with laser sintering forming thin conductive traces. No evident reduction of the LED brightness was observed under $80 \%$ strain. Reproduced with permission, ${ }^{61}$ copyright 2021. American Chemical Society. (g) Direct writing of PVP based LM nano-ink on various substrates. Reproduced with permission, ${ }^{52}$ copyright 2021, Elsevier.

suspended tannic acid molecules is carried out. After synthesis, a printing ink containing a high concentration of EGaIn nanoparticles suspended in TA solution is created for writing conductive traces on various rigid or soft surfaces by using a ballpoint pen as presented in Fig. 10e.

Other methods to form conductive traces for printed electronics involve printing LM nanodroplets with a grafted polymer layer in order to enable adhesion to diverse substrates while adjusting ink's viscosity. The formulated ink can have double core-shell structured LM nanoparticles with an oxide and polydimethylsiloxane (PDMS) shell (i.e., double emulsion ink). ${ }^{61}$ To create this double emulsion ink, bulk EGaIn is probe sonicated in ethanol followed by PDMS precursors. The second step allows the PDMS to be attached to the ethanol-oxide interface to form double grafted emulsions of the LM nanoparticles. By using this processing technique, the double emulsion ink can contain a high loading of LM ( $98.4 \%$ by weight), while having low viscosity $(6 \times$ larger than water), large shear thinning behavior, and high surface wetting characteristics for an efficient printing process. Finally, the double emulsion LM particles can form stretchable ( $\sim 80 \%$ strain) conductive traces with a resolution up to $37 \mu \mathrm{m}$ when selectively sintered by laser irradiation (Fig. 10f). Thus, through surface modification, the polymer coating on LM nanoparticles helps to minimize the ink viscosity and improves surface wettability for rapid printing of soft electronics.

To create a water-based LM nano-ink, the synthesized LM nanoparticles are grafted with polyvinylpyrrolidone (PVP), a water-soluble and biocompatible stabilizer, to ensure dispersion stability of the particles. ${ }^{52}$ The LM nano-ink can be filled into a ballpoint pen and can print conductive traces with various configurations and on various organic or inorganic substrates (Fig. 10g). These conductive traces from LM nanoparticles can also be casted on filter paper, laser patterned, and encapsulated in PDMS to make flexible antennas and capacitors with their resistance stable against bending or stretching. There is also an effort to produce a LM printing ink that enables recyclability, printability, and compatibility with various surfaces. For instance, EGaIn microdroplets (70 to $100 \mu \mathrm{m})$ coated with a water-soluble poly(vinyl alcohol) (PVA) polymer form a LM ink 
wettable on various surfaces (i.e., polyacrylamide hydrogel, poly(ethylene terephthalate) and polyurethane), exhibit shear thinning behaviors and are recoverable as bulk EGaIn when dissolved in sodium hydroxide. ${ }^{53}$ This ink can form functional conductive traces for electronic skins to detect various body movements including pressing, bending, stretching, and even subtle airflows.

\subsection{Flexible energy harvesting devices}

Liquid metal composites with embedded micro or nanosized particles have been used as thermal interfaces and electrical interconnections for stretchable and wearable thermoelectric energy generators (TEG). ${ }^{30,106,107}$ The use of nano-/microparticles enables the suppression of freezing and melting points of LM droplets compared to those of bulk LM. When this freezing point is lower than the glass transition temperature of the encapsulating polymer, the LM composite can remain soft and stretchable even at extremely low temperatures as the LM inclusions remain as a fluid. Moreover, this suppressed freezing point or supercooling properties of LM micro/nanoparticles occur independently of the type of encapsulating polymers (Fig. 11a). Thus, this enables a broad range of LM polymer composites to remain compliant at extreme low temperatures, in the range of $-85{ }^{\circ} \mathrm{C}$. Taking advantage of this property, this LM composite is used as a compliant thermal interface of a wearable TEG for harnessing body heat under cold conditions to electrically power an electronic sleeve (Fig. 11b) in addition to powering a wearable pulse oximeter sensor. ${ }^{30}$ Since LM composites can also form conductive traces by selective mechanical sintering, they can serve as both a thermal conductor and an electrical interconnector. ${ }^{106}$ With these combined functionalities, the LM composite can be used to fabricate soft and stretchable thermoelectric devices by replacing the conventional stiff interconnectors such as copper and silver with stretchable activated traces of LM particles. In a more recent study, a LM elastomer composite with enhanced thermal conductivity was used as a thermal interface material to fabricate not only flexible but also stretchable thermoelectric generator as shown in Fig. 11c.

Smaller liquid metal particles in elastomer composites prevent the leakage of LM at high volume fractions, which may cause undesirable LM contact with the skin and the formation of short circuit within the device. However, reducing the particle size to the nanoscale level can stiffen the LM-PDMS
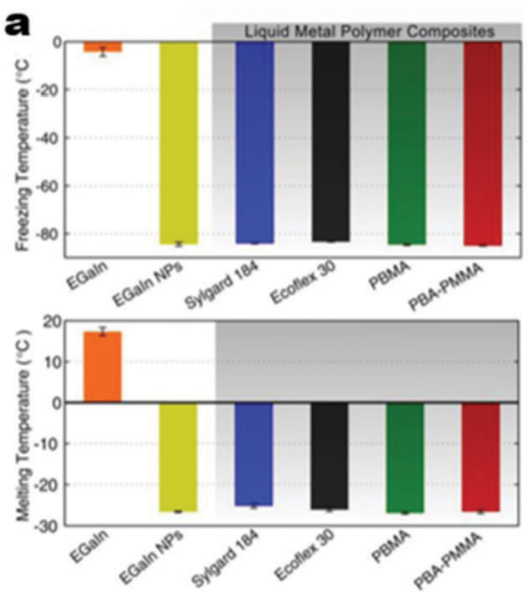

d

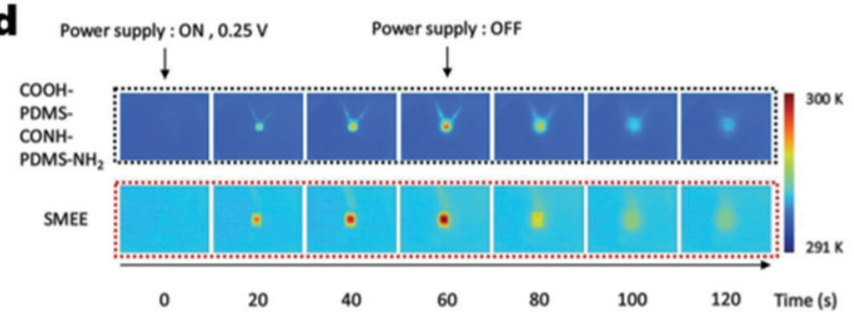

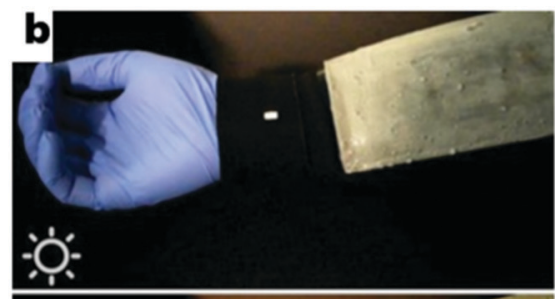
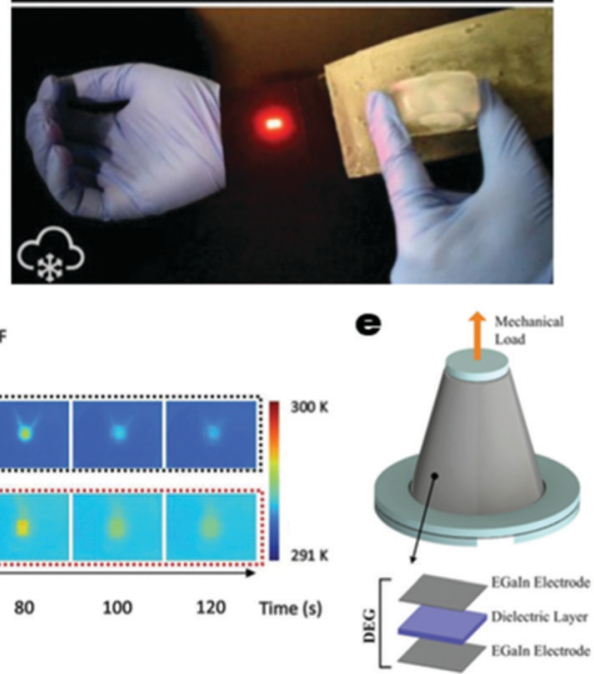
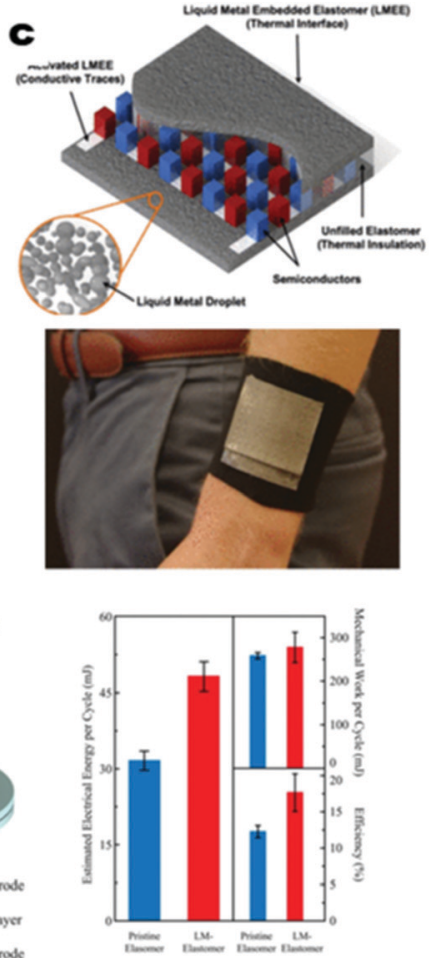

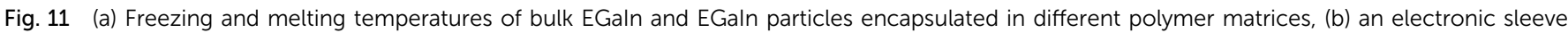

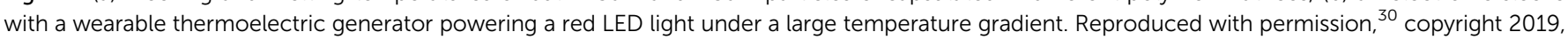

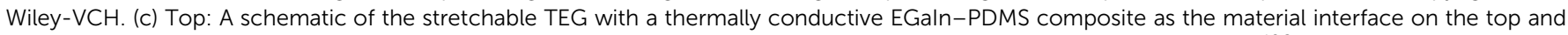

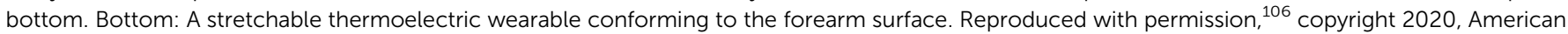

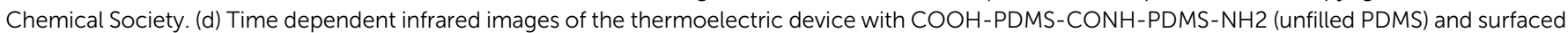

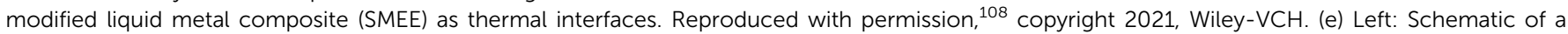

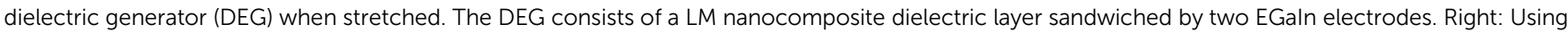

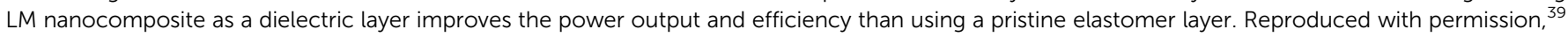
copyright 2019, Wiley-VCH. 
composites with limited improvement of their thermal conductivity because of low LM volume fraction $(<20 \%)$ in the nanocomposite. Thus, to reap the benefits of LMNPs while maximizing thermal conductivity, high volume fractions of LMNPs should be embedded in the polymer matrix without a significant effect on the composite's stiffness. A recent work overcomes this challenge by modifying the surface of LMNPs with carboxylic acid terminated PDMS to fabricate a stretchable LM nanocomposite with up to $44 \%$ filler volume fraction and with reasonable stretchability $(110 \%$ strain break; $350 \%$ for unfilled polymer). ${ }^{108}$ With this novel LM nanocomposite, a stretchable and thermally conductive thermal interface can be demonstrated to enhance the temperature gradient across a unit cell thermoelectric generator during active heating or cooling as shown in Fig. 11d. Another use of LM nanodroplets is the production of a stretchable high dielectric material for fabricating stretchable dielectric energy generators with high power conversion efficiency. ${ }^{39}$ This is possible because nanoscale LM inclusions can increase the effective dielectric constant of the LM composites with a moderate decrease in compliance. Synthesized liquid metal nanoparticles have also been successfully downsized into quantum dots for solar energy harvesting. ${ }^{74}$ It is shown that these liquid metal quantum dots ( 5 to $9 \mathrm{~nm}$ in diameter) can enhance the ultraviolet absorption efficiency of perovskite solar cells with improved power conversion by $17 \%$. To date, there is no substantial work on LM nanoparticle application for solar energy harvesting, but it is expected to receive further attention in the future developments of flexible wearables integrated with solar energy harvesting modules.

\subsection{LMNPs in nanomedicine}

Liquid metal nanoparticles are advantageous in the field of nanomedicine due to their reduced cytotoxicity, small size for easy intracellular transport, and high photothermal energy conversion at the interface. ${ }^{64}$ These features enable LM nanoparticles to function as agents for rapid DNA detection and therapeutic carriers with light-controllable drug release for targeted treatment of cancer cells.

For rapid DNA detection, gallium nanoparticles are incorporated into a biomedical device to detect a specific gene mutation associated with cystic fibrosis from a DNA sequence. ${ }^{109}$ This device consists of Ga plasmonic nanoparticles that are deposited on silicon substrates by a thermal evaporation method. The result is a device with a formed pseudo dielectric platform that functions as a selective DNA biosensing layer that can detect a targeted gene sequence even in the presence of impurities (pathogen materials). For instance, this DNA detection platform can selectively detect the presence of a single nucleotide polymorphism of a specific genetic sequence of Helicobacter pylori, the gene mutation indicative of cystic fibrosis. In addition, this fabrication technique is demonstrated to be versatile and can be tailored to detect a variety of other mutated genes with high sensitivity. Hence, these DNA sensing systems can be very useful over previous gene detection procedures by providing rapid and targeted gene detection from a DNA sample.
Highly shape transformable LMNPs with attached therapeutics have also been proven effective in aiding intracellular drug delivery. ${ }^{44}$ These functional liquid metal nanoparticles are surface modified with graphene quantum dots (GQDs) by sonication before being grafted with doxorubicin (DOX) drug molecules. GQDs on the interface of LM nanoparticles act as high energy collectors to convert light to heat energy which aids in changing the shape of the spherical nanoparticles to hollow rods. During this light-induced shape transformation, the attached drug molecules are controllably released within the targeted cell. To study the efficacy of this treatment mechanism, this study examines the antitumor efficacy when the LMNPs with DOX are injected into HeLa tumor-bearing mice followed by near-infrared light irradiation at the tumor site (Fig. 12a). The mice that were injected with the liquid metal nanoparticles showed strong inhibition efficacy to tumor growth, with the tumors eliminated completely by day 14 (Fig. 12b). Even without the GQD surface deposition, the LM nanoparticles with a biocompatible surface polymer coating (with polyethylene glycol functional group) exhibit a strong photothermal effect for controllable drug delivery by shape transformation from nanospheres to nanocapsules as shown in Fig. $12 \mathrm{c}$ and $\mathrm{d}^{43}$ Moreover, similar shape transformable LMNPs can be used to enhance X-ray imaging in biological organs and living mice for cancer cell diagnostic purposes (Fig. 12e).

A more recent study in this area has demonstrated that LMNPs not only induce cancer cell death but also prevent cancer cell recurrence. By creating a locally injectable LM doxorubicin nanoflake hydrogel nanocomposite, an enhanced prevention of postoperative tumor relapse can be achieved compared to just using LMNPs with doxorubicin attachment (Fig. 12f). ${ }^{110}$ Instead of using laser irradiation, these LM nanoflakes use $\mathrm{pH}$ changes as the stimuli to initiate drug release. The nanoflake structure of synthesized LM nanodroplets enables a larger loading capacity of DOX therapeutic by $63.5 \%$ compared to previous LM-based nanospheres and nanorods. To create this nanostructure, surface functionalization of LMNPs with hyperbranched poly(amidoamine) ligand is conducted by sonication. Further encapsulation with hydrogel delays the DOX drug release and is the key approach to achieve a long-term antitumor effect and lower drug toxicity at the targeted cancer treatment site. As a result, the LM nanoflakes synthesized in this way have the potential to be utilized as a post-surgical cancer treatment to decrease the chance of relapsing tumors. These studies have demonstrated the emerging use of liquid metal micro-/nanoparticles in biomedical applications. Additional studies should be conducted to ensure the safety of LMNPs and their composites for long term applications.

\section{Summary}

In this review article, we summarized recent developments in the synthesis and applications of liquid metal nanoparticles. From the synthesis perspective, mechanical shearing and sonication are two current attractive methodologies for the rapid 

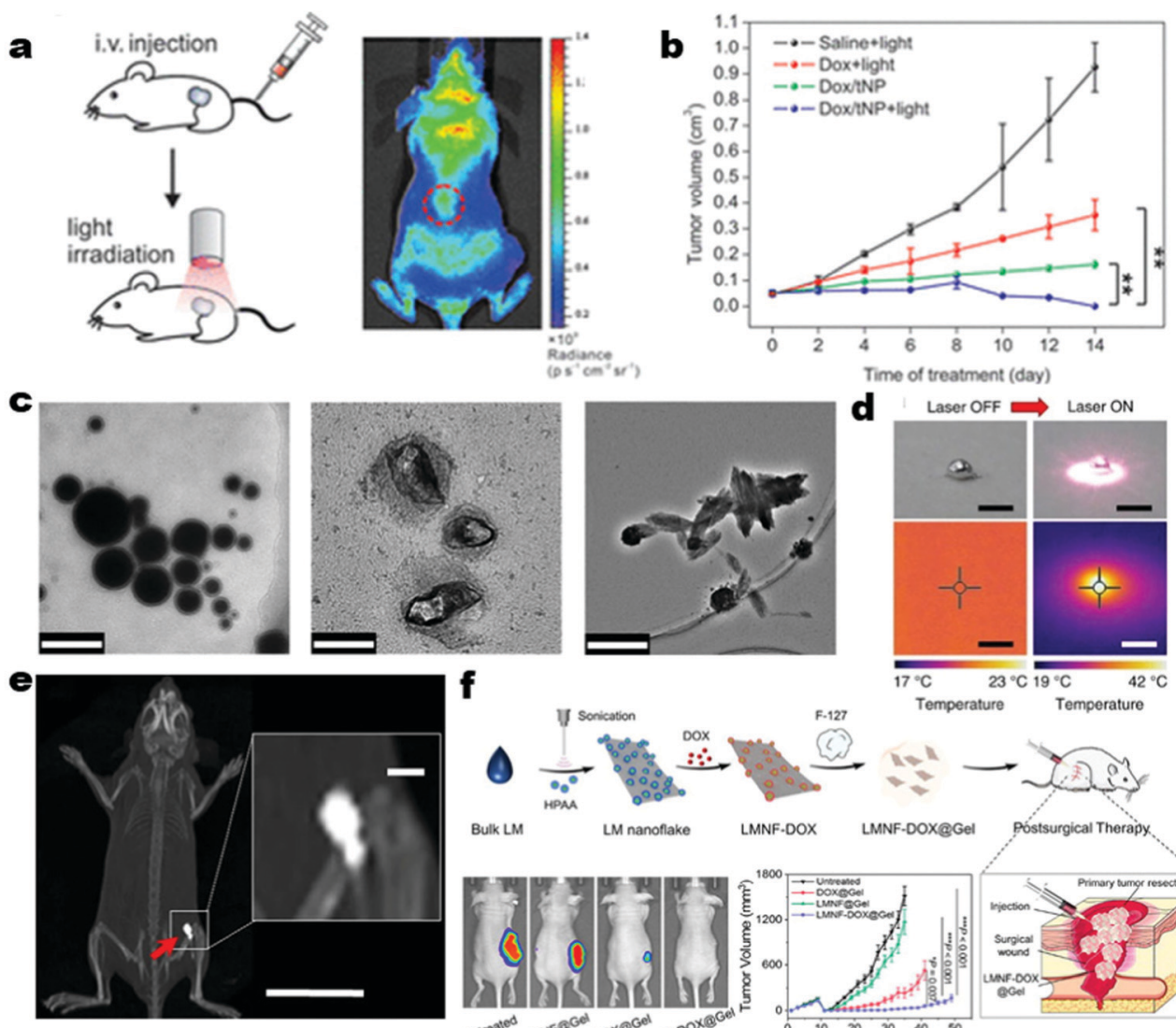

d
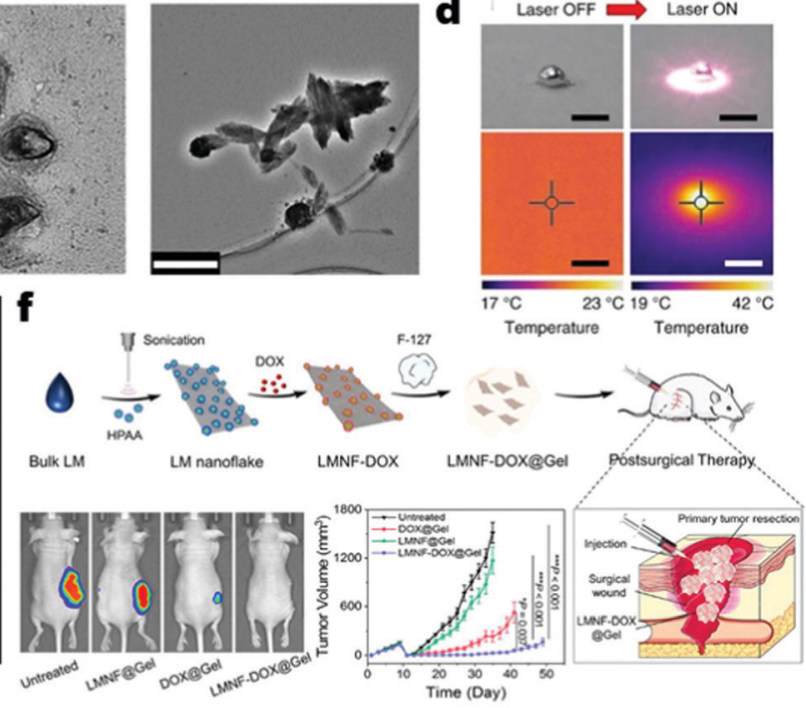

Fig. 12 (a) Schematic of HeLa tumor-bearing mice injected with saline, free DOX solution, and DOX on transformable LM nanoparticles. Light treatment groups were irradiated with light after the injection. (b) Diseased tissue is circled in red and HeLa tumor growth curves through 14 days. Reproduced with permission, ${ }^{44}$ copyright 2017, American Chemical Society. (c) TEM images of LM nanocapsules before laser irradiation, after 1 minute of laser irradiation, and after 3 minutes of laser irradiation. Scale bars represents $200 \mathrm{~nm}, 100 \mathrm{~nm}$, and $500 \mathrm{~nm}$ labelled from left to right, (d) visible light and thermographic images of LM droplet before and after laser irradiation from a $785 \mathrm{~nm}$ near infrared laser at $1 \mathrm{~W}$ for $5 \mathrm{~min}$, (e) 3D-X-ray image of LM nanocapsules injected in living mouse. The red arrow displays the laser-irradiated site with a magnified view of laser-irradiated part. Scale bar, $3 \mathrm{~cm}$ (inset: $2 \mathrm{~mm}$ ). Reproduced with permission, ${ }^{43}$ copyright 2017 , Nature. (f) Schematic of LM nanoflake hydrogel formulation for post-surgical cancer treatment. Reproduced with permission, ${ }^{110}$ copyright 2021 , Elsevier.

synthesis of LM micro/nanoparticles because of their ability to control the size of particles while allowing surface modification. Mechanical shearing with or without an impeller allows facile and scalable synthesis of LM micro-/nanodroplets and nanocomposites with average sizes down to several microns by varying the mixing speed, time, and LM-solvent volume ratios. When a large yield of LMNPs is desired, probe- or bath-based ultrasonication can be used to generate LM droplets with an average size of several tenths of nanometers. Successful synthesis of LM nanoparticles via sonication relies on optimized sonication power, time, concentration, and type of surfactants.

When bulk LM is reduced to nanoscale, a large surface area of the oxide layer is present, offering diverse opportunities for chemical functionalization or modification. In summary, these strategies consist of coating organic and inorganic materials on the oxide shell. Other solutions involve chemically modifying the surface chemistry or initiating the cross-linking of monomers (i.e., surface-initiated polymerization) on the surface of $\mathrm{Ga}$ oxide to either substitute the Ga oxide layer with resilient ligands or creating an additional reinforcing monolayer on the formed LM nanoparticles. The preferred surface modification on LMNPs will depend on their functional applications such as enhancing their surface adhesion properties for additive manufacturing of stretchable conductors, interface tuning for LM-polymer nanocomposites, or enhancing photothermal conversion efficiency for nanomedicine. Concurrently, a concentrated effort has also been made to incorporate LM nanoparticles as stretchable and functional nanocomposites in wearable energy harvesting devices. New studies are also expected in the photovoltaic systems in order to leverage the metallic properties of LMNPs and their high stability. Based on this summary, there are still fundamental questions yet to be answered to enable reliable synthesis of LM nanoparticles in large quantities with their morphologies and functional properties customizable for desired applications.

\section{Outlook and future directions}

Generating monodispersed liquid metal nanoparticles by emulsion shearing and sonication remains a formidable challenge despite the emergence of various innovative interface tuning 
strategies. A synergistic processing technique involving microfluidic and sonication seems to produce relatively narrow size distribution of droplets but is unable to create $<100 \mathrm{~nm}$ nanoparticles with high yield. Extensive study on the relationship between processing parameters and final size distribution and morphology of LMNPs has been made. However, more investigation should be done to better understand how the interaction between these processing parameters affects the properties (both chemical and mechanical) of the interface and the final macroscale properties of the nanocomposites. Another research direction is exploring universal surface engineering solutions for LMNPs that will ensure colloidal stability in both organic and aqueous media while having diverse attributes such as surface-independent adhesion properties or particlematrix/solvent compatibility. Once the processing method of LM nanoparticles has matured, automated synthesis can eventually replace in lab synthesis to allow scalable, safe, rapid, and reproducible quality of the generated LM nanoparticles. The automated synthesis approach will involve an on demand endto-end continuous fabrication featuring large-scale emulsion shearing and sonication processing for synthesizing LMNPs while monitoring the physical and chemical properties in real time.

Elemental gallium is produced mainly as a by-product making its production and availability limited unlike other abundant elements. Therefore, practical strategies to recycle and reuse Ga-based LMNPs should be considered. A few studies had shown that soluble coating on LMNPs can be removed while the surface oxidation is reversible in highly basic and acidic medium. In this way, bulk LM can be collected and reused. For LM nanocomposites, having soluble polymer matrices is essential to successfully recover the embedded LMNPs. For the most part, the recovery of bulk LM requires hazardous chemicals, is limited to certain nanocomposites, and requires special chemical treatments. Hence, to ensure sustainable application of LMNPs, more studies should be done on developing cost-effective and green chemistry methods to recycle and reuse LM from synthesized LMNPs and LM nanocomposites after reaching their end of use.

To achieve commercialization, the reliability and long-term stability of LM nanocomposite need to be verified. For instance, extensive investigations need to be performed to reveal the stability of the dielectric constant of LM nanocomposites over time and under different conditions such as high loading cycles, high electric field and at extreme temperatures. Similar studies should also be conducted for identifying the stability of electrical and thermal properties of the composites. In parallel, an effort to identify the best interface-tuning approach to ensure durability and stability of properties of the nanocomposite is critical for future commercialization.

Limited knowledge on the reactivity, corrosiveness and cytotoxicity of liquid metal nanoparticles remains a concern for their incorporation in wearable and biomedical applications. Recent studies have hypothesized that the large surface area of passivating $\mathrm{Ga}$ oxide layer on LM nanoparticles suppress the formation of $\mathrm{Ga}^{3+}$ ions which reduces their reactivity when in close contact with other metals or tissue cells. More in depth investigation into these proposed mechanisms and additional low cytotoxicity evidence need to be presented to convince future stakeholders on the long-term safety of Ga-based LMNPs and to continue their growth in biomedical applications.

As the functionalities and applications of synthesized liquid metal nanoparticle broaden, advanced modeling tools guided by experimental measurements should be taken advantage to predict the mechanical and functional properties of the LM nanocomposites or nanoparticles. Finite element methods and micromechanics modeling have been useful for understanding and predicting the mechanical, thermal, electrical, and coupling behaviors of LM composites at the continuum level. However, these modeling techniques rely on simplifying assumptions at the oxide interface such as the bonding conditions, size, thermal, mechanical, and electrical properties of the oxide shell. To accurately predict the effects of various surface engineering on the bulk properties of LM composites, multiscale modeling approaches that combine molecular dynamics (MD) simulation at the interface and continuum computational model at the mesoscale should be synergized to accelerate the design of functional LMNPs or nanocomposites while reducing experimental time. In addition, MD and density functional theory computations can aid in simulating chemical processes at the interface to explore novel ligand molecules or surface engineering processes that can enhance the multifunctional properties of LMNPs.

Overall, we foresee that as the interest and number of researchers who are actively studying nanoscale liquid metals continue to increase, we expect to see more interdisciplinary problem-solving approaches emerging in the future along with more compelling applications of liquid metal nanoparticles and nanocomposites.

\section{Conflicts of interest}

There are no conflicts to declare.

\section{References}

1 R. C. Chiechi, E. A. Weiss, M. D. Dickey and G. M. Whitesides, Eutectic gallium-indium (EGaIn): A moldable liquid metal for electrical characterization of selfassembled monolayers, Angew. Chem., Int. Ed., 2008, 47(1), 142-144, DOI: 10.1002/anie.200703642.

2 B. Kim, et al., Interfacing liquid metals with stretchable metal conductors, ACS Appl. Mater. Interfaces, 2015, 7(15), 7920-7926, DOI: 10.1021/am508899z.

3 A. Miner and U. Ghoshal, Cooling of high-power-density microdevices using liquid metal coolants, Appl. Phys. Lett., 2004, 85, 506, DOI: 10.1063/1.1772862.

4 X.-D. Zhang, et al., Experimental investigation of galinstan based minichannel cooling for high heat flux and large 
heat power thermal management, Energy Convers. Manage, 2019, 185(1), 248-258, DOI: 10.1016/j.enconman.2019.02.010.

5 J. H. Kim, S. Kim, J. H. So, K. Kim and H. J. Koo, Cytotoxicity of Gallium-Indium Liquid Metal in an Aqueous Environment, ACS Appl. Mater. Interfaces, 2018, 10(20), 17448-17454, DOI: 10.1021/acsami.8b02320.

6 Y. Plevachuk, V. Sklyarchuk, S. Eckert, G. Gerbeth and R. Novakovic, Thermophysical properties of the liquid Ga-In-Sn eutectic alloy, J. Chem. Eng. Data, 2014, 465, 1-9, DOI: 10.1021/je400882q.

7 D. Zrnic and D. S. Swatik, On the resistivity and surface tension of the eutectic alloy of gallium and indium, J. Less-Common Met., 1969, 18(1), 67-68, DOI: 10.1016/0022-5088(69)90121-0.

8 M. H. Malakooti, M. R. Bockstaller, K. Matyjaszewski and C. Majidi, Liquid metal nanocomposites, Nanoscale Adv., 2020, 2(7), 2668-2677, DOI: 10.1039/D0NA00148A.

9 S. Chen, H. Z. Wang, R. Q. Zhao, W. Rao and J. Liu, Liquid Metal Composites, Matter, 2020, 2(6), 1446-1480, DOI: 10.1016/j.matt.2020.03.016.

10 K. Kalantar-Zadeh, et al., Emergence of Liquid Metals in Nanotechnology, ACS Nano, 2019, 13(7), 7388-7395, DOI: 10.1021/acsnano.9b04843.

11 Z. J. Farrell and C. Tabor, Control of Gallium Oxide Growth on Liquid Metal Eutectic Gallium/Indium Nanoparticles via Thiolation, Langmuir, 2018, 34(1), 234-240, DOI: 10.1021/acs.langmuir.7b03384.

12 T. Daeneke, et al., Liquid metals: Fundamentals and applications in chemistry, Chem. Soc. Rev., 2018, 47(11), 4073-4111, DOI: 10.1039/c7cs00043j.

13 Y. Yang, S. Sun, S.-Y. Tang, W. Li and S. Zhang, Viscoelastic Properties of Gallium-indium Alloy, Appl. Rheol., 2018, 28, 42903, DOI: 10.3933/ApplRheol-28-42903.

14 M. R. Khan, C. B. Eaker, E. F. Bowden and M. D. Dickey, Giant and switchable surface activity of liquid metal via surface oxidation, Proc. Natl. Acad. Sci. U. S. A., 2014, 111, 14047-14051, DOI: 10.1073/pnas.1412227111.

15 C. Ladd, J. H. So, J. Muth and M. D. Dickey, 3D printing of free standing liquid metal microstructures, Adv. Mater., 2013, 25(36), 5081-5085, DOI: 10.1002/adma.201301400.

16 F. C. Simeone, et al., Defining the Value of Injection Current and Effective Electrical Contact Area for EGaInBased Molecular Tunneling Junctions, J. Am. Chem. Soc., 2013, 135(48), 18131-18144, DOI: 10.1021/ja408652h.

$17 \mathrm{~J}$. Chen, et al., Understanding interface (odd-even) effects in charge tunneling using a polished EGaIn electrode, Phys. Chem. Chem. Phys., 2018, 20(7), 4864-4878, DOI: 10.1039/C7CP07531F.

18 S. Park, S. Kang and H. J. Yoon, Power Factor of One Molecule Thick Films and Length Dependence, ACS Cent. Sci., 2019, 5(12), 1975-1982, DOI: 10.1021/acscentsci.9b01042.

19 S. Park and H. J. Yoon, New Approach for Large-Area Thermoelectric Junctions with a Liquid Eutectic GalliumIndium Electrode, Nano Lett., 2018, 18(12), 7715-7718, DOI: $10.1021 /$ acs.nanolett.8b03404.

20 S. Park, J. Jang and H. J. Yoon, Validating the Mott Formula with Self-Assembled Monolayer (SAM)-Based Large-Area
Junctions: Effect of Length, Backbone, Spacer, Substituent, and Electrode on the Thermopower of SAMs, J. Phys. Chem. C, 2021, 125(36), 20035-20047, DOI: 10.1021/acs. jpcc.1c05623.

21 R. Rao, K. G. Eyink and B. Maruyama, Single-walled carbon nanotube growth from liquid gallium and indium, Carbon, 2010, 48(13), 3971-3973, DOI: 10.1016/j.carbon.2010.06.065.

22 M. Mousavi, et al., Bismuth telluride topological insulator synthesized using liquid metal alloys: Test of NO2 selective sensing, Appl. Mater. Today, 2021, 22, 100954, DOI: 10.1016/j.apmt.2021.100954.

$23 \mathrm{H}$. Khan, et al., Liquid metal-based synthesis of high performance monolayer SnS piezoelectric nanogenerators, Nat. Commun., 2020, 11(1), 3449, DOI: 10.1038/s41467-02017296-0.

24 N. Syed, A. Zavabeti and J. Ou, et al., Printing twodimensional gallium phosphate out of liquid metal, Nat. Commun., 2018, 9(1), 3618, DOI: 10.1038/s41467-018-06124-1.

25 J. N. Hohman, et al., Directing Substrate Morphology via Self-Assembly: Ligand-Mediated Scission of GalliumIndium Microspheres to the Nanoscale, Nano Lett., 2011, 11(12), 5104-5110, DOI: 10.1021/nl202728j.

26 Y. Lin, Y. Liu, J. Genzer and M. D. Dickey, Shapetransformable liquid metal nanoparticles in aqueous solution, Chem. Sci., 2017, 8(5), 3832-3837, DOI: 10.1039/ C7SC00057J.

27 Y. Liu, W. Zhang and H. Wang, Synthesis and Application of Core-Shell Liquid Metal Particles: A Perspective of Surface Engineering, Mater. Horiz., 2020, 8(1), 56-77, DOI: $10.1039 / \mathrm{D} 0 \mathrm{MH} 01117 \mathrm{G}$.

28 W. Zhang, et al., Liquid Metal/Metal Oxide Frameworks, Adv. Funct. Mater., 2014, 24(24), 3799-3807, DOI: 10.1002/ adfm.201304064.

29 L. Ren, et al., Nanodroplets for Stretchable Superconducting Circuits, Adv. Funct. Mater., 2016, 26(44), 8118, DOI: 10.1002/adfm.201603427.

30 M. H. Malakooti, et al., Liquid Metal Supercooling for Low-Temperature Thermoelectric Wearables, Adv. Funct. Mater., 2019, 29(45), 1906098, DOI: 10.1002/adfm.201906098.

31 A. Yamaguchi, Y. Mashima and T. Iyoda, Reversible Size Control of Liquid-Metal Nanoparticles under Ultrasonication, Angew. Chem., Int. Ed., 2015, 54(43), 12809-12813, DOI: 10.1002 /anie.201506469.

32 Y. Lin, et al., Sonication-enabled rapid production of stable liquid metal nanoparticles grafted with poly(1-octadecenealt-maleic anhydride) in aqueous solutions, Nanoscale, 2018, 10(42), 19871-19878, DOI: 10.1039/C8NR05600E.

33 A. Fassler and C. Majidi, Liquid-Phase Metal Inclusions for a Conductive Polymer Composite, Adv. Mater., 2015, 27(11), 1928-1932, DOI: 10.1002/adma.201405256.

34 M. D. Bartlett, et al., Stretchable, High- k Dielectric Elastomers through Liquid-Metal Inclusions, Adv. Mater., 2016, 28(19), 3726-3731, DOI: 10.1002/adma.201506243.

35 E. J. Markvicka, M. D. Bartlett, X. Huang and C. Majidi, An autonomously electrically self-healing liquid metalelastomer composite for robust soft-matter robotics and 
electronics, Nat. Mater., 2018, 17(7), 618-624, DOI: 10.1038/ s41563-018-0084-7.

36 I. D. Tevis, L. B. Newcomb and M. Thuo, Synthesis of Liquid Core-Shell Particles and Solid Patchy Multicomponent Particles by Shearing Liquids Into Complex Particles (SLICE), Langmuir, 2014, 30(47), 14308-14313, DOI: 10.1021/la5035118.

37 S. Çınar, I. D. Tevis, J. Chen and M. Thuo, Mechanical Fracturing of Core-Shell Undercooled Metal Particles for Heat-Free Soldering, Sci. Rep., 2016, 6(1), 21864, DOI: 10.1038/srep21864.

38 R. Tutika, S. Kmiec, A. B. M. T. Haque, S. W. Martin and M. D. Bartlett, Liquid Metal-Elastomer Soft Composites with Independently Controllable and Highly Tunable Droplet Size and Volume Loading, ACS Appl. Mater. Interfaces, 2019, 11(19), 17873-17883, DOI: 10.1021/acsami.9b04569.

39 C. Pan, et al., A Liquid-Metal-Elastomer Nanocomposite for Stretchable Dielectric Materials, Adv. Mater., 2019, 31(23), 1900663, DOI: 10.1002/adma.201900663.

40 J. J. Chang, A. Martin, C. Du, A. M. Pauls and M. Thuo, Heat-Free Biomimetic Metal Molding on Soft Substrates, Angew. Chem., Int. Ed., 2020, 59(38), 16346-16351, DOI: 10.1002/anie.202008621.

41 A. Martin, et al., Heat-Free Fabrication of Metallic Interconnects for Flexible/Wearable Devices, Adv. Funct. Mater., 2019, 29(40), 1903687, DOI: 10.1002/adfm.201903687.

42 J. Yan, et al., Shape-controlled synthesis of liquid metal nanodroplets for photothermal therapy, Nano Res., 2019, 12(6), 1313-1320, DOI: 10.1007/s12274-018-2262-y.

43 S. A. Chechetka, et al., Light-driven liquid metal nanotransformers for biomedical theranostics, Nat. Commun., 2017, 8(1), 15432, DOI: 10.1038/ncomms15432.

44 Y. Lu, et al., Enhanced Endosomal Escape by Light-Fueled Liquid-Metal Transformer, Nano Lett., 2017, 17(4), 2138-2145, DOI: 10.1021/acs.nanolett.6b04346.

45 L. Castilla-Amorós, D. Stoian, J. R. Pankhurst, S. B. Varandili and R. Buonsanti, Exploring the chemical reactivity of gallium liquid metal nanoparticles in galvanic replacement, J. Am. Chem. Soc., 2020, 142(45), 1928319290, DOI: 10.1021/jacs.0c09458.

46 P. Fan, et al., Nano liquid metal for the preparation of a thermally conductive and electrically insulating material with high stability, RSC Adv., 2018, 8(29), 16232-16242, DOI: $10.1039 / \mathrm{c} 8 \mathrm{ra00262b.}$

47 J. Mingear, Z. Farrell, D. Hartl and C. Tabor, Galliumindium nanoparticles as phase change material additives for tunable thermal fluids, Nanoscale, 2021, 13(2), 730-738, DOI: 10.1039/D0NR06526A.

48 R. W. Style, R. Tutika, J. Y. Kim and M. D. Bartlett, SolidLiquid Composites for Soft Multifunctional Materials, $A d v$. Funct. Mater., 2021, 31(1), 2005804, DOI: 10.1002/adfm. 202005804.

49 C. Chiew and M. H. Malakooti, A Double Inclusion Model for Liquid Metal Polymer Composites, Compos. Sci. Technol., 2021, 208, 108752, DOI: 10.1016/j.compscitech. 2021.108752.
50 M. J. Ford, M. Palaniswamy, C. P. Ambulo, T. H. Ware and C. Majidi, Size of liquid metal particles influences actuation properties of a liquid crystal elastomer composite, Soft Matter, 2020, 16(25), 5878-5885, DOI: 10.1039/ D0SM00278J.

51 J. W. Boley, E. L. White and R. K. Kramer, Mechanically Sintered Gallium-Indium Nanoparticles, Adv. Mater., 2015, 27(14), 2355-2360, DOI: 10.1002/adma.201404790.

52 M. Zhang, et al., Versatile fabrication of liquid metal nanoink based flexible electronic devices, Appl. Mater. Today, 2021, 22, 100903, DOI: 10.1016/j.apmt.2020.100903.

$53 \mathrm{~J} . \mathrm{Xu}$, et al., Printable and Recyclable Conductive Ink Based on a Liquid Metal with Excellent Surface Wettability for Flexible Electronics, ACS Appl. Mater. Interfaces, 2021, 13(6), 7443-7452, DOI: 10.1021/acsami.0c20549.

54 M. A. Rahim, et al., Polyphenol-Induced Adhesive Liquid Metal Inks for Substrate-Independent Direct Pen Writing, Adv. Funct. Mater., 2021, 31(10), 2007336, DOI: 10.1002/ adfm.202007336.

55 T. R. Lear, et al., Liquid metal particle popping: Macroscale to nanoscale, Extreme Mech. Lett., 2017, 13, 126-134, DOI: 10.1016/j.eml.2017.02.009.

56 N. J. Morris, Z. J. Farrell and C. E. Tabor, Chemically modifying the mechanical properties of core-shell liquid metal nanoparticles, Nanoscale, 2019, 11(37), 17308-17318, DOI: 10.1039/C9NR06369B.

57 Y. Liu, X. Ji and J. Liang, Rupture stress of liquid metal nanoparticles and their applications in stretchable conductors and dielectrics, npj Flexible Electron., 2021, 5(1), 11, DOI: 10.1038/s41528-021-00108-w.

58 J.-E. Park, et al., Rewritable, Printable Conducting Liquid Metal Hydrogel, ACS Nano, 2019, 13(8), 9122-9130, DOI: 10.1021/acsnano.9b03405.

59 S. Liu, D. S. Shah and R. Kramer-Bottiglio, Highly stretchable multilayer electronic circuits using biphasic galliumindium, Nat. Mater., 2021, 20(6), 851-858, DOI: 10.1038/ s41563-021-00921-8.

60 S. Liu, et al., Laser Sintering of Liquid Metal Nanoparticles for Scalable Manufacturing of Soft and Flexible Electronics, ACS Appl. Mater. Interfaces, 2018, 10(33), 28232-28241, DOI: 10.1021/acsami.8b08722.

61 S. Liu, S. Y. Kim, K. E. Henry, D. S. Shah and R. KramerBottiglio, Printed and Laser-Activated Liquid MetalElastomer Conductors Enabled by Ethanol/PDMS/Liquid Metal Double Emulsions, ACS Appl. Mater. Interfaces, 2021, 13(24), 28729-28736, DOI: 10.1021/acsami.0c23108.

62 H. Song, et al., Ga-Based Liquid Metal Micro/Nanoparticles: Recent Advances and Applications, Small, 2020, 16(12), 1903391, DOI: 10.1002/smll.201903391.

63 Y. Lin, J. Genzer and M. D. Dickey, Attributes, Fabrication, and Applications of Gallium-Based Liquid Metal Particles, Adv. Sci., 2020, 7(12), 2000192, DOI: 10.1002/advs.202000192.

64 H. Li, R. Qiao, T. P. Davis and S.-Y. Tang, Biomedical Applications of Liquid Metal Nanoparticles: A Critical Review, Biosensors, 2020, 10(12), 196, DOI: 10.3390/bio s10120196. 
65 A. Martin, B. S. Chang, A. M. Pauls, C. Du and M. Thuo, Stabilization of Undercooled Metals via Passivating Oxide Layers, Angew. Chem., Int. Ed., 2021, 60(11), 5928-5935, DOI: 10.1002/anie.202013489.

66 A. Martin, C. Du, A. M. Pauls, T. Ward III and M. Thuo, Polydispersity-Driven Printing of Conformal Solid Metal Traces on Non-Adhering Biological Surfaces, Adv. Mater. Interfaces, 2020, 7(22), 2001294, DOI: 10.1002/admi. 202001294.

67 B. Abismaïl, J. Canselier, A. Wilhelm, H. Delmas and C. Gourdon, Emulsification by ultrasound: drop size distribution and stability, Ultrason. Sonochem., 1999, 6(1-2), 75-83, DOI: 10.1016/S1350-4177(98)00027-3.

68 Y. Lin, et al., Handwritten, Soft Circuit Boards and Antennas Using Liquid Metal Nanoparticles, Small, 2015, 11(48), 6397-6403, DOI: 10.1002/smll.201502692.

69 H. Lu, et al., Dynamic Temperature Control System for the Optimized Production of Liquid Metal Nanoparticles, ACS Appl. Nano Mater., 2020, 3(7), 6905-6914, DOI: 10.1021/ acsanm.0c01257.

70 J. He, F. Shi, J. Wu and J. Ye, Shape Transformation Mechanism of Gallium-Indium Alloyed Liquid Metal Nanoparticles, Adv. Mater. Interfaces, 2021, 8(6), 2001874, DOI: $10.1002 /$ admi.202001874.

71 S. Tang, et al., Functional Liquid Metal Nanoparticles Produced by Liquid-Based Nebulization, Adv. Mater. Technol., 2019, 4(2), 1800420, DOI: 10.1002/admt.201800420.

72 S. Tang, et al., Microfluidic Mass Production of Stabilized and Stealthy Liquid Metal Nanoparticles, Small, 2018, 14(21), 1800118, DOI: 10.1002/smll.201800118.

73 G. Bo, et al., Gallium-Indium-Tin Liquid Metal Nanodroplet-Based Anisotropic Conductive Adhesives for Flexible Integrated Electronics, ACS Appl. Nano Mater., 2021, 4(1), 550-557, DOI: 10.1021/acsanm.0c02870.

$74 \mathrm{~S}$. Li, et al., Laser induced core-shell liquid metal quantum dots for high-efficiency carbon-based perovskite solar cells, Appl. Surf. Sci., 2021, 565, 150470, DOI: 10.1016/j.apsusc. 2021.150470.

75 N. Kazem, T. Hellebrekers and C. Majidi, Soft Multifunctional Composites and Emulsions with Liquid Metals, Adv. Mater., 2017, 29(27), 1605985, DOI: 10.1002/adma.201605985.

76 N. Kazem, M. D. Bartlett and C. Majidi, Extreme Toughening of Soft Materials with Liquid Metal, Adv. Mater., 2018, 30(22), 1706594, DOI: 10.1002/adma.201706594.

77 H. Wang, et al., A Highly Stretchable Liquid Metal Polymer as Reversible Transitional Insulator and Conductor, Adv. Mater., 2019, 31(23), 1901337, DOI: 10.1002/adma. 201901337.

78 M. J. Ford, D. K. Patel, C. Pan, S. Bergbreiter and C. Majidi, Controlled Assembly of Liquid Metal Inclusions as a General Approach for Multifunctional Composites, Adv. Mater., 2020, 32(46), 2002929, DOI: 10.1002/adma.202002929.

79 R. J. Larsen, M. D. Dickey, G. M. Whitesides and D. A. Weitz, Viscoelastic properties of oxide-coated liquid metals, J. Rheol., 2009, 53(6), 1305-1326, DOI: 10.1122/ 1.3236517 .
80 K. B. Ozutemiz, J. Wissman, O. B. Ozdoganlar and C. Majidi, EGaIn-Metal Interfacing for Liquid Metal Circuitry and Microelectronics Integration, Adv. Mater. Interfaces, 2018, 5(10), 1701596, DOI: 10.1002/admi.201701596.

81 J. Wang, S. Liu, S. Guruswamy and A. Nahata, Reconfigurable liquid metal based terahertz metamaterials via selective erasure and refilling to the unit cell level, Appl. Phys. Lett., 2013, 103(22), 221116, DOI: 10.1063/1.4837675.

82 M. R. Khan, C. Trlica, J.-H. So, M. Valeri and M. D. Dickey, Influence of Water on the Interfacial Behavior of Gallium Liquid Metal Alloys, ACS Appl. Mater. Interfaces, 2014, 6(24), 22467-22473, DOI: 10.1021/am506496u.

$83 \mathrm{~J}$. Yan, et al., Solution processable liquid metal nanodroplets by surface-initiated atom transfer radical polymerization, Nat. Nanotechnol., 2019, 14(7), 684-690, DOI: 10.1038/ s41565-019-0454-6.

84 Q. Wei, et al., Cu-Catalyzed Atom Transfer Radical Polymerization in the Presence of Liquid Metal Micro/Nanodroplets, Macromolecules, 2021, 54(4), 1631-1638, DOI: 10.1021/acs.macromol.0c02702.

85 Y. Su, G. Sui, J. Lan and X. Yang, A highly stretchable dielectric elastomer based on core-shell structured soft polymer-coated liquid-metal nanofillers, Chem. Commun., 2020, 56(78), 11625-11628, DOI: 10.1039/D0CC04224B.

86 Q. Wei, et al., Surface Engineering of Liquid Metal Nanodroplets by Attachable Diblock Copolymers, ACS Nano, 2020, 14(8), 9884-9893, DOI: 10.1021/acsnano.0c02720.

87 M. Fromel, M. Li and C. W. Pester, Surface Engineering with Polymer Brush Photolithography, Macromol. Rapid Commun., 2020, 41(18), 2000177, DOI: 10.1002/marc.2020 00177.

88 C. Zhang, et al., Nucleation and Growth of Polyaniline Nanofibers onto Liquid Metal Nanoparticles, Chem. Mater., 2020, 32(11), 4808-4819, DOI: 10.1021/acs.chemmater. 0c01615.

89 S. Veerapandian, et al., Hydrogen-doped viscoplastic liquid metal microparticles for stretchable printed metal lines, Nat. Mater., 2021, 20(4), 533-540, DOI: 10.1038/s41563-02000863-7.

90 J. Ma, et al., Liquid Metal Nanoparticles as Initiators for Radical Polymerization of Vinyl Monomers, ACS Macro Lett., 2019, 8(11), 1522-1527, DOI: 10.1021/acsmacro lett.9b00783.

91 X. Li, et al., Liquid Metal Initiator of Ring-Opening Polymerization: Self-Capsulation into Thermal/Photomoldable Powder for Multifunctional Composites, Adv. Mater., 2020, 32(43), 2003553, DOI: 10.1002/adma.202003553.

92 T. Gan, et al., Liquid Metal-Mediated Mechanochemical Polymerization, Macromol. Rapid Commun., 2019, 40(24), 1900537, DOI: 10.1002/marc.201900537.

93 B. Fan, J. Wan, Y. Liu, W. W. Tian and S. H. Thang, Functionalization of liquid metal nanoparticles via the RAFT process, Polym. Chem., 2021, 12(20), 3015-3025, DOI: 10.1039/D1PY00257K.

94 Z. J. Farrell, C. J. Thrasher, A. E. Flynn and C. E. Tabor, Silanized Liquid-Metal Nanoparticles for Responsive 
Electronics, ACS Appl. Nano Mater., 2020, 3(7), 6297-6303, DOI: 10.1021 /acsanm.0c01056.

95 Z. J. Farrell, N. Reger, I. Anderson, E. Gawalt and C. Tabor, Route to Universally Tailorable Room-Temperature Liquid Metal Colloids via Phosphonic Acid Functionalization, J. Phys. Chem. C, 2018, 122(46), 26393-26400, DOI: 10.1021/ acs.jpcc.8b07042.

96 N. Ilyas, A. Cook and C. E. Tabor, Designing Liquid Metal Interfaces to Enable Next Generation Flexible and Reconfigurable Electronics, Adv. Mater. Interfaces, 2017, 4(15), 1700141, DOI: 10.1002/admi.201700141.

97 M. A. Creighton, et al., Oxidation of Gallium-based Liquid Metal Alloys by Water, Langmuir, 2020, 36(43), 12933-12941, DOI: 10.1021/acs.langmuir.0c02086.

98 Y. Liu, et al., Water-processable liquid metal nanoparticles by single-step polymer encapsulation, Nanoscale, 2020, 12(25), 13731-13741, DOI: 10.1039/D0NR00988A.

99 Y. Liu, Q. Wang, J. Deng and W. Zhang, A liquid metal composite by ZIF-8 encapsulation, Chem. Commun., 2020, 56(12), 1851-1854, DOI: 10.1039/C9CC09330C.

100 T. Gan, W. Shang, S. Handschuh-Wang and X. Zhou, LightInduced Shape Morphing of Liquid Metal Nanodroplets Enabled by Polydopamine Coating, Small, 2019, 15(9), 1804838, DOI: 10.1002/smll.201804838.

101 M. A. Creighton, M. C. Yuen, N. J. Morris and C. E. Tabor, Graphene-based encapsulation of liquid metal particles, Nanoscale, 2020, 12(47), 23995-24005, DOI: 10.1039/ D0NR05263A.

102 K. Khoshmanesh, et al., Liquid metal enabled microfluidics, Lab Chip, 2017, 17(6), 974-993, DOI: 10.1039/C7LC00046D.
103 L. Zhu, et al., Liquid Metal-Based Soft Microfluidics, Small, 2020, 16(9), 1903841, DOI: 10.1002/smll.201903841.

104 J. W. Boley, E. L. White, G. T. C. Chiu and R. K. Kramer, Direct Writing of Gallium-Indium Alloy for Stretchable Electronics, Adv. Funct. Mater., 2014, 24(23), 3501-3507, DOI: $10.1002 /$ adfm.201303220.

105 F. Centurion, et al., Liquid metal dispersion by selfassembly of natural phenolics, Chem. Commun., 2019, 55(75), 11291-11294, DOI: 10.1039/C9CC06081B.

106 M. Zadan, M. H. Malakooti and C. Majidi, Soft and Stretchable Thermoelectric Generators Enabled by Liquid Metal Elastomer Composites, ACS Appl. Mater. Interfaces, 2020, 12(15), 17921-17928, DOI: 10.1021/acsami.9b19837.

107 M. Zadan, C. Chiew, C. Majidi and M. H. Malakooti, Liquid Metal Architectures for Soft and Wearable Energy Harvesting Devices, Multifunct. Mater., 2020, 4(1), 012001, DOI: 10.1088/2399-7532/abd4f0.

108 H. Bark, M. W. M. Tan, G. Thangavel and P. S. Lee, Deformable High Loading Liquid Metal Nanoparticles Composites for Thermal Energy Management, Adv. Energy Mater., 2021, 11(35), 2101387, DOI: 10.1002/aenm.2021 01387.

109 A. G. Marín, et al., Gallium plasmonic nanoparticles for label-free DNA and single nucleotide polymorphism sensing, Nanoscale, 2016, 8(18), 9842-9851, DOI: 10.1039/ C6NR00926C.

$110 \mathrm{~J}$. Xiong, et al., Injectable liquid metal nanoflake hydrogel as a local therapeutic for enhanced postsurgical suppression of tumor recurrence, Chem. Eng. J., 2021, 416, 129092, DOI: 10.1016/j.cej.2021.129092. 